\title{
Evaluating the Performance of the PC-SAFT and CPA Equations of State on Anomalous Properties of Water
}

Tsochantaris, Evangelos; Liang, Xiaodong; Kontogeorgis, Georgios M.

Published in:

Journal of Chemical and Engineering Data

Link to article, DOI:

10.1021/acs.jced.0c00689

Publication date:

2020

Document Version

Peer reviewed version

Link back to DTU Orbit

Citation (APA):

Tsochantaris, E., Liang, X., \& Kontogeorgis, G. M. (2020). Evaluating the Performance of the PC-SAFT and CPA Equations of State on Anomalous Properties of Water. Journal of Chemical and Engineering Data, 65(12), 5718-5734. https://doi.org/10.1021/acs.jced.0c00689

\section{General rights}

Copyright and moral rights for the publications made accessible in the public portal are retained by the authors and/or other copyright owners and it is a condition of accessing publications that users recognise and abide by the legal requirements associated with these rights.

- Users may download and print one copy of any publication from the public portal for the purpose of private study or research.

- You may not further distribute the material or use it for any profit-making activity or commercial gain

- You may freely distribute the URL identifying the publication in the public portal 


\title{
Evaluating the performance of the PC-SAFT and CPA equations of state on anomalous properties of water
}

\author{
Evangelos Tsochantaris ${ }^{\dagger}$, Xiaodong Liang $^{\dagger}$, Georgios Kontogeorgis ${ }^{\dagger *}$ \\ ${ }^{\dagger}$ Center for Energy Resources Engineering (CERE) \\ Department of Chemical and Biochemical Engineering \\ Technical University of Denmark \\ DK-2800, Kongens Lyngby \\ Denmark \\ *corresponding author e-mail: gk@kt.dtu.dk
}

\begin{abstract}
Water is the most important and at the same time the most anomalous substance on earth. Due to its importance, there are numerous studies concerned with the modeling of water using advanced equations of state (EOS) like those based on Wertheim's perturbation theory. However, only few of these studies deal explicitly with the performance of these models in predicting water's anomalous properties. In this study, the performance of the perturbed chainstatistical associating fluid theory (PC-SAFT) and the cubic-plus-association (CPA) EOS on predicting several water's properties is investigated. Twelve PC-SAFT and two CPA parameter sets from literature are used and compared. Despite an overall acceptable performance of both models, all parameter sets fail to satisfactorily describe second-order derivative properties and there is not a parameter set that is clearly superior. None of the two models is also clearly better than the other. Most importantly, none of the parameter sets is able to accurately predict water's anomalous properties like the maximum of density or minimum of heat capacity with respect to temperature. These results indicate that significant improvements in the models and their underlying theories are needed for the accurate description of water's complex thermodynamic behavior. The most promising approach is to take into consideration water's structure.
\end{abstract}

Keywords: Water, Anomalies, Thermodynamics, Equations of state, Statistical mechanics

\section{Introduction}

Water is the most important substance in the world; it covers two thirds of the Earth and our own cells include two-thirds water by volume. Hundreds of books have been written about water, yet we know so little about it. In the words of Philip Ball, consultant of Nature: "No one really understands water. It's still a mystery"1. In fact, water is the most anomalous substance on earth, exhibiting more than 50 exceptional properties. These properties have important implications for engineering, chemistry, biology and medicine. Professor Chaplin's web-site provides a good collection of these "anomalous" water properties ${ }^{2}$. Among the most noteworthy ones are the maximum of density at $4{ }^{\circ} \mathrm{C}$ (ice floats, lakes freeze from top to bottom), high values of heat capacity (thus stabilizing Earth's climate) and surface tension (small insects walk on water) and the maxima and minima of many thermodynamic properties as function of temperature, e.g. the 
minimum hydrocarbon solubility in water at room temperature (related to the hydrophobic effect), see figure 1.

Predicting the unique behavior of water can be challenging for any theoretical model. Among the possible approaches, equations of state (EOS) have played a pivotal role in the calculation of phase equilibria and various thermodynamic properties of fluids. Classical cubic equations of state have been used extensively in industry because of their simplicity and relative accuracy ${ }^{3,4}$. However, they are inadequate for substances containing strongly associating molecules, such as water ${ }^{4}$. Thus, more complex or advanced, molecular EOS, in which molecular association is accounted for explicitly, should be advantageous ${ }^{4}$. Some of the most popular molecular EOS are those based on the Wertheim's perturbation theory ${ }^{5-8}$, which have received acceptance in academia and -to some extent- also industry, because of their accuracy and wide application range ${ }^{4}$.

It is by now widely known that such advanced thermodynamic models can represent well very complex phase equilibria e.g. water-hydrocarbon liquid-liquid equilibria ${ }^{9}$. This is shown in figure 1 where three advanced models are used. All three models shown in figure 1 can predict thermodynamic behavior for many aqueous systems by modeling water as a four-site associating compound. None of the models displayed in this figure can represent the minimum in hydrocarbon solubility at low temperatures.

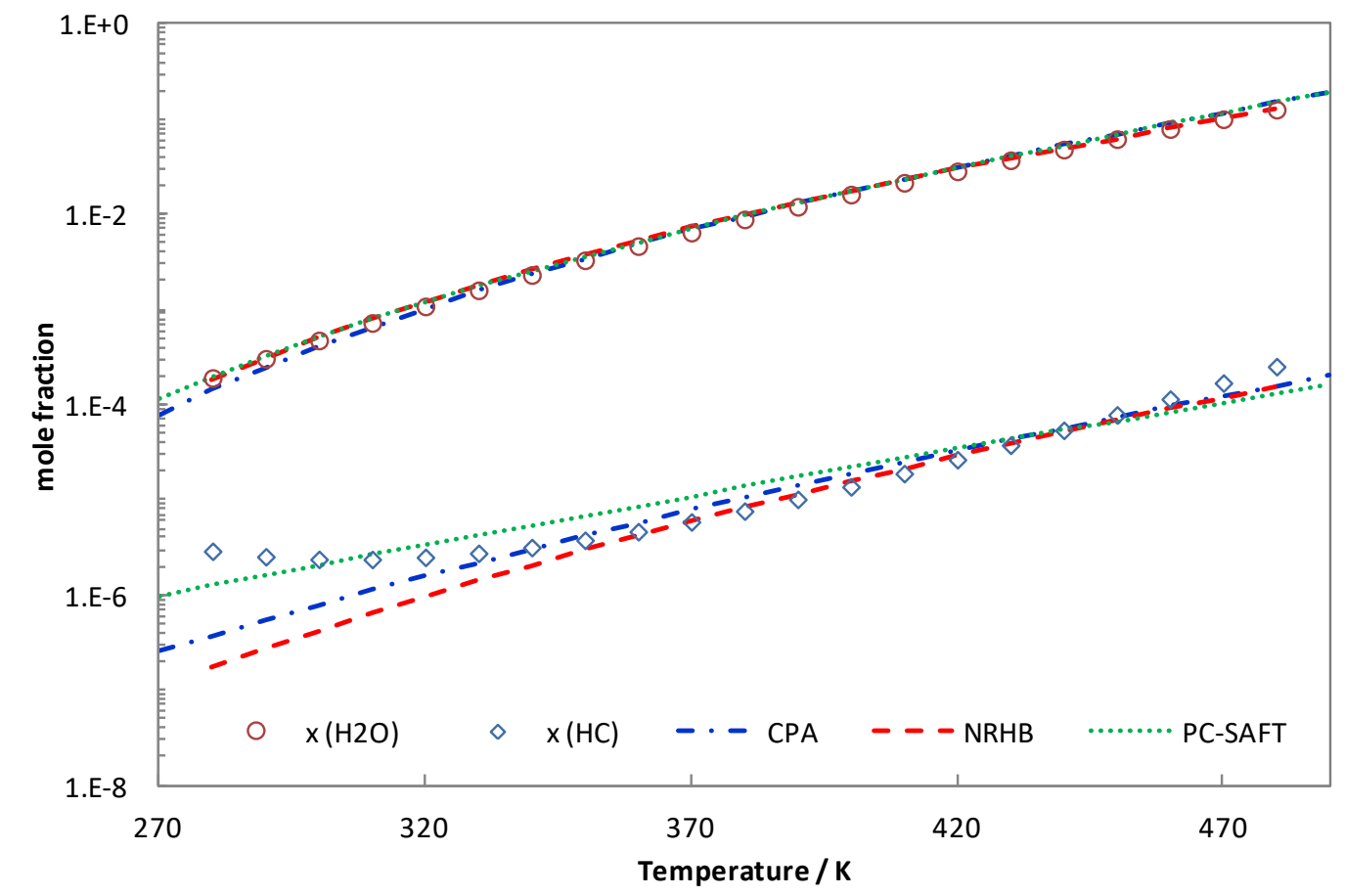

Figure 1. Liquid-liquid equilibria of water-hexane with three thermodynamic models. All models use a single adjustable parameter. Reproduced with permission from ref. 9. Copyright 2016 Elsevier.

Despite the fact that these models account for hydrogen bonding and the fact that they have shown great accuracy at predicting phase equilibria of various aqueous mixtures, the extent in which they are able to predict water's properties in not entirely clear. As shown in figure 2, none of the three models mentioned here is in agreement with the spectroscopic data of water previously 
measured by Luck ${ }^{10}$. The molecular simulation data ${ }^{11}$ and the dielectric constant theory by MariboMogensen et al. ${ }^{12}$ agree with the three models but again not with the experimental data.

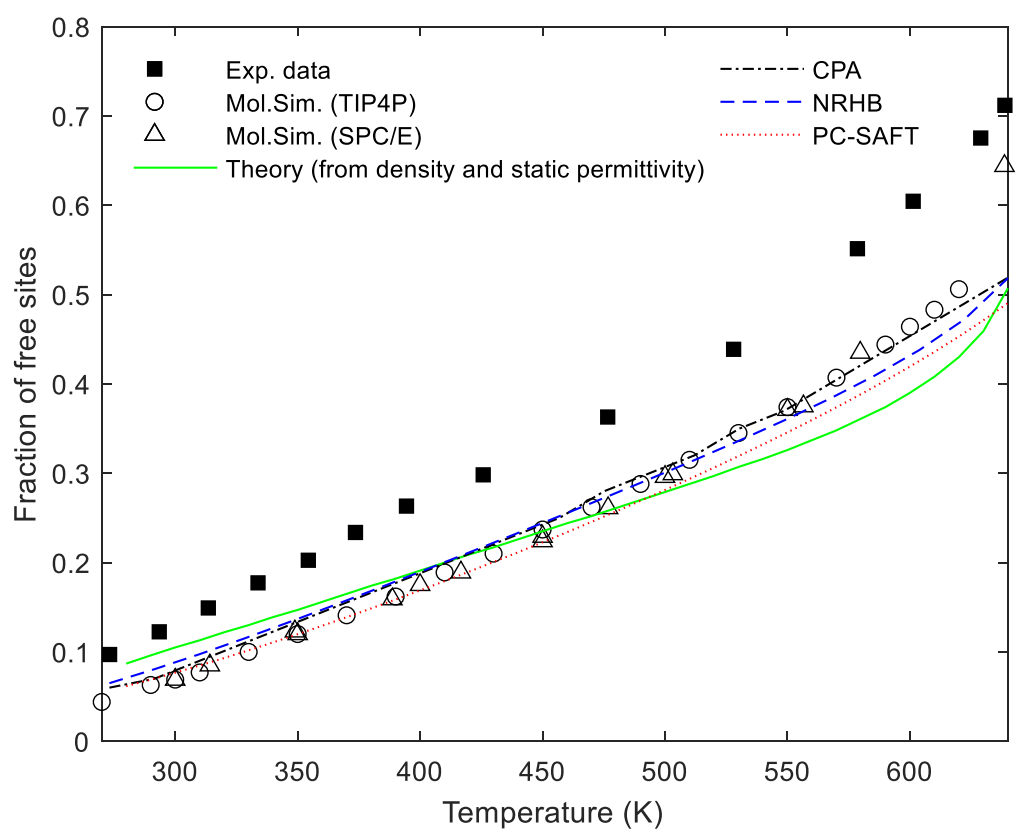

Figure 2. Free site fraction of saturated water using thermodynamic models, experimental data, a new theory and recent molecular simulation data. Water is assumed to have four association sites with all models. Reproduced with permission from ref. 9. Copyright 2016 Elsevier.

Monomer fraction data for water have been used by many authors in connection with association theories. Some of these studies are works of Müller and Gubbins ${ }^{13}$, Aparicio-Martinez and $\mathrm{Hall}^{3}$, Clark et al. ${ }^{14}$, von Solms et al. ${ }^{15}$, Grenner et al. ${ }^{16}$, Kontogeorgis et al. ${ }^{17}$, Vega and Llovell $^{18}$ and Marshall ${ }^{19}$. In fact some studies, like Clark et al. ${ }^{14}$ and Marshall ${ }^{19}$ have managed to describe the monomer fraction data with greater accuracy than the models illustrated in Figure 2. In other words many studies have used Luck's data ${ }^{10}$ for evaluating their model's performance, but new recent monomer fraction data ${ }^{20}$ of water are in disagreement with Luck's data ${ }^{10}$ suggesting higher amounts of free water molecules (see also discussion by Liang et al. ${ }^{9}$ ). Additionally, there is no consensus on the amount of association sites that should be attributed to water (see also section 3). The large amount of studies attempting to model water along with the conflicting views indicate that our understanding of water is lacking.

In this article, two advanced thermodynamic models (CPA and PC-SAFT) are applied for the prediction of pure liquid water's properties with an emphasis on its unusual thermodynamic behavior. It should be noted that there are many studies on molecular EOS that have modeled water. Most of them focus on the prediction of phase equilibria of aqueous mixtures that are useful for industrial applications and few studies attempt to predict derivative properties like heat capacity or speed of sound. For the purposes of our research, we have investigated the models' performance for vapor pressure, density, isobaric heat capacity, isochoric heat capacity and speed of sound over a wide range of temperatures and pressures. 


\section{Thermophysical properties of water}

Water has many anomalies that are usually connected to its physical properties, like the density maximum with respect to temperature. The true extent of these anomalies is not yet known and currently there are not any scientific studies that have attempted to list all of them. Professor Martin Chaplin in his website ${ }^{2}$ has attempted to achieve this feat where he lists many water's anomalies. He lists 74 anomalies out of which approximately 24 are connected to thermodynamic properties of liquid water. In this section, two of the thermodynamic properties of water are presented in order to illustrate their unusual behavior. These properties are density and residual isobaric heat capacity. For the investigation of these properties, experimental data have been collected over a wide range of temperatures and pressures. In addition to the experimental data, calculated values from the NIST Webbook ${ }^{21}$ are used. The NIST Webbook ${ }^{21}$ is using the "IAPWS formulation 1995 formulation for the thermodynamic properties of ordinary water substance" 22 to generate these values and although they are not experimental data, these values are expected to be very close to the experimental ones. The IAPWS-95 formulation ${ }^{22}$ is an empirical model designed to predict pure water's thermodynamic properties. Results from this model are going to be referred as results from the NIST model ${ }^{21}$ since they were acquired from the NIST Webbook ${ }^{21}$.

As mentioned previously, one well known anomaly of water is the density maximum at $4{ }^{\circ} \mathrm{C}$ $(277 \mathrm{~K})$ and atmospheric pressure $(0.1 \mathrm{MPa})$. What is less-known is the fact that this maximum shifts with pressure (Figure 3). In fact at high pressures (>200 MPa) there no longer seems to be a maximum. Results from the NIST model are very close to the experimental values, which is to be expected since all of these data have also been used for the development of the IAPWS-95 formulation $^{22}$. 

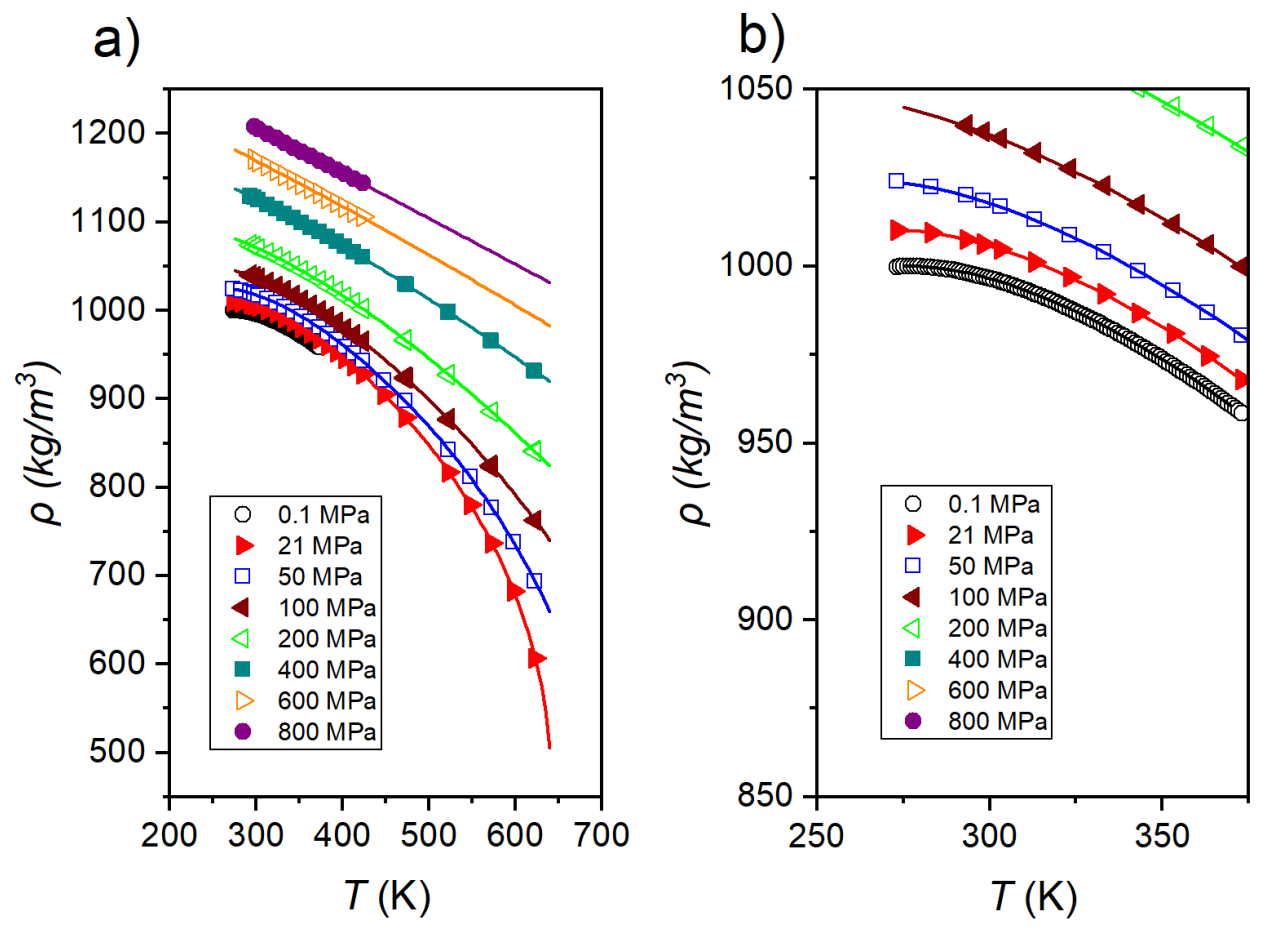

Figure 3. Density of liquid water as a function of temperature and pressure a) Plot of all the collected values b) Enlarged graph to emphasize the density maximum. The symbols represent the experimental data ${ }^{23-26}$. The lines represent the results from the NIST model ${ }^{21}$. The estimated uncertainty of the experimental data ranges from $\pm 0.02 \%$ to $\pm 0.2 \%$. More information on the estimated uncertainties can be obtained from the original studies ${ }^{23-26}$. An extensive analysis of all the collected data ${ }^{23-26}$ is available in the study for the development of IAPWS-95 formulation ${ }^{21}$.

Another thermodynamic property of water that shows unusual behavior is the residual isobaric heat capacity $C_{P}^{r}$. As shown in Figure 4 , there is a minimum at around $310 \mathrm{~K}$ and $P=0.1 \mathrm{MPa}$. However, like the density maximum, this minimum no longer exists at higher pressures, at which region the residual isobaric heat capacity is becoming more important ${ }^{27}$. Moreover, as shown in both Figures $4 \mathrm{a}$ and $4 \mathrm{~b}$, there is a complete change in the curvature of the isobars to the point that maxima appear in the highest pressure regions. It is not certain whether these maxima are anomalies since there might be other compounds that could exhibit this behavior. It is worth noting that the experimental data shown in Figure 4 were available at 2011, many years after the development of the IAPWS-95 formulation, and at that time, there was limited amount of $C_{P}^{r}$ data for liquid water for pressures above $100 \mathrm{MPa}$. Even so, the values from NIST are extremely close to the experimental ones. The original publication of the experimental data ${ }^{28}$ also contains more detailed comparisons to the values from NIST.

From these two properties, it is clear that water has a complex thermodynamic behavior unlike any other pure substance. At the same time, some similarities between the thermodynamic properties can be observed. The properties tend to display extrema when plotted against temperature and the behavior of every property seems to be strongly pressure dependent. In this study, more thermodynamic properties have been investigated (e.g. isochoric heat capacity and speed of sound) and their special characteristics are discussed at section 5 . 
a)

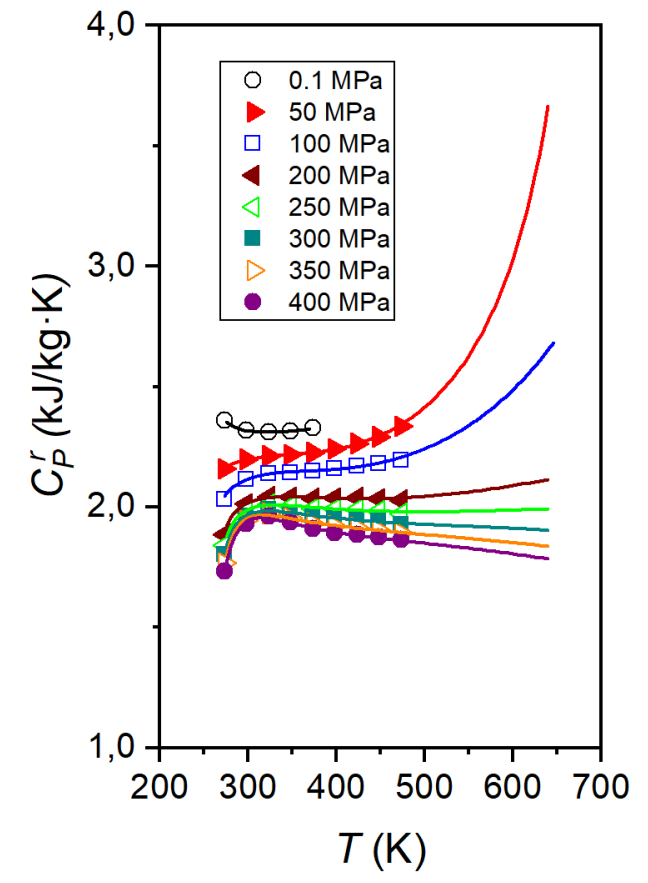

b)

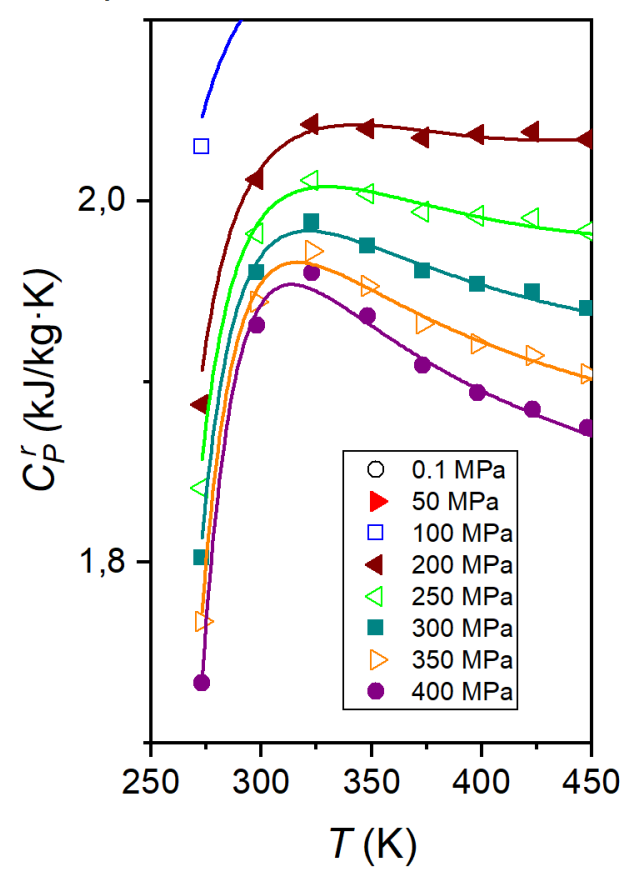

Figure 4. Residual isobaric heat capacity of liquid water as a function of temperature and pressure. a) Graph of all collected experimental data ${ }^{28}$ b) Enlarged graph to emphasize the maxima. The symbols represent the data calculated from experimental values of isobaric heat capacity ${ }^{28}$ and ideal gas heat capacity values from DIPPR ${ }^{29}$. The lines represent predicted values calculated from values of isobaric heat capacity provided by the NIST model ${ }^{21}$ and ideal gas heat capacity values from DIPPR ${ }^{29}$. The estimated uncertainty of the experimental data is $\pm 0.2 \%$. More information on the estimated uncertainties can be obtained from the original study ${ }^{28}$.

\section{Review of some thermodynamic models applied to water}

Since water is the most common substance in earth, the modelling studies of water are abundant. This study focuses on molecular EOS, i.e. equations of states that are firmly rooted on statistical mechanics and are explicitly accounting for hydrogen bonding. Two such equations are the "perturbed chain-SAFT" (PC-SAFT) and the "cubic-plus-association" (CPA) EOS. They are briefly described in the next section. Both of these EOS require five pure component parameters to be specified for each associating fluid. Three of these parameters are needed in the physical term to describe the molecule segment diameter or co-volume, number of segments (PC-SAFT) and dispersion energy. The remaining two parameters characterize the hydrogen bonding sites on the molecule. The CPA equation of state has a similar association term as PC-SAFT, but the two models describe repulsive and dispersion interactions differently ${ }^{15}$, see section 4 .

Water containing systems have been studied with the PC-SAFT equation of state for years and more than twenty sets of pure component parameters have been published for water that focus on different applications ${ }^{30}$. Aqueous systems have been also studied extensively with CPA using 
various association schemes, but only few of these sets are used in most studies. It is evident that the modelling of these systems has attracted the interest of many researchers. At the same time, the modelling of pure water and other water containing systems has proven to be quite challenging, especially if a very wide range of properties and conditions are considered. Within the SAFT framework, water has been modelled as a two-site (2B), three-site (3B) and four-site (4C) molecule, but it is not entirely clear which approach is the most suitable ${ }^{4,30}$. Some authors claim that the three-site model is more accurate suggesting that only three hydrogen bonds can be formed due to steric hindrance ${ }^{31}$, while others ${ }^{32,33}$ claim that the four-site model provides more accurate predictions ${ }^{4}$.

Even though there are many parameter sets available for water and there has been a considerable amount of research in order to predict liquid-liquid equilibria (LLE) or vapor-liquid equilibria (VLE) of aqueous mixtures, there have not been many studies focusing explicitly on the prediction of water's anomalous properties. Of course, the accurate prediction of VLE and LLE is of crucial importance to many industrial applications. However, an investigation about the performance of these equations of state for these special anomalous properties of water could provide insight on whether these models are general enough or sufficient for describing the complex thermodynamic behavior of water. In this work, twelve PC-SAFT parameter sets and two CPA parameter sets are evaluated for their capabilities in predicting several of pure liquid water's thermodynamic properties. A short review of literature follows. In this short review, we will mostly discuss about the application of these models for pure water and water containing mixtures. For the application of PC-SAFT and CPA on aqueous mixtures one should also take into account interaction parameters, mixing rules and potentially cross-association of different molecules. This study is focused on pure water's thermodynamic properties and thus our discussion will not be extended to the interaction parameters or mixing rules that are required to model mixtures.

Gross and Sadowski ${ }^{34}$ applied the PC-SAFT EOS to systems in which molecular association prevails including pure components and mixtures. They tested the performance of the model for alkanols, amines, acetic acid and water and pure component parameter sets for these compounds are published. They assumed two association sites for all compounds (including water) and the pure component parameters were obtained by simultaneously fitting vapor pressure and saturated liquid density data. For water, they also modeled the phase equilibria of the system water-1pentanol. Deviations of $1.88 \%$ for vapor pressure and $6.83 \%$ were observed for pure water (see also Section 5). The system of water-1-pentanol shows a liquid-liquid equilibrium at lower temperatures and a heteroazeotropic vapor-liquid equilibrium. The results of the model showed some deviations from the vapor-liquid data, but the results for the liquid-liquid equilibrium were very accurate.

Cameretti et al. ${ }^{35}$ extended the PC-SAFT EOS by adding a term from the Debye-Hückel theory $^{36}$ for modelling electrolyte solutions and the new EOS is called ePC-SAFT. This term represents the contribution to the Helmholtz free energy for charging up a hard sphere system. For this modified equation of state, one would still need to assign values to the pure component parameters of water. The pure component parameters of water were adjusted simultaneously to vapor pressure and saturated liquid density data at the $T$ range of 278-393 K (see also Section 5). There was an excellent agreement between the calculated values and the experimental values of vapor pressure and saturated liquid density. The new ePC-SAFT EOS was able to accurately predict the vapor pressure and liquid density of single-salt solutions as well as of mixed salt solutions. Some of the salts that were included in the study were $\mathrm{NaCl}, \mathrm{LiCl}, \mathrm{KCl}, \mathrm{NaBr}, \mathrm{LiBr}$, and $\mathrm{KBr}$. 
The performance of PC-SAFT in predicting monomer fraction of water was investigated by von Solms et al. ${ }^{15}$. In their study, they compared different parameter sets for water (from literature and own values) and attempted to take into account the physical meaning of the pure component parameters without sacrificing the accuracy of vapor pressure and saturated liquid density predictions. Their new parameter sets were obtained by fixing the chain length $m$ at equally spaced values ranging from $m=2.00$ to $m=3.50$ while allowing the remaining four parameters to vary and they assumed four association sites (4C) for water. All studied sets of parameters for PC-SAFT were acceptable in predicting vapor pressure and liquid density data. In order to evaluate the performance of the different parameter sets, they also took into account monomer fraction data. The sets were able to predict monomer fraction of water reasonably well and it was concluded that the $4 \mathrm{C}$ scheme is the most suitable for water based on their results. However, at this point it should be mentioned that the monomer fraction data used were the ones from Luck ${ }^{10}$. As discussed in Section 1, there also more recent monomer fraction data published by Mallamace et al. ${ }^{20}$ that contradict Luck's data ${ }^{10}$.

Grenner et al. ${ }^{16}$ studied with PC-SAFT different literature parameter sets for water, while also introducing a new one. For assigning values to the parameters, they took into account the physical meaning of the pure component parameters while fitting to vapor pressure and saturated liquid density data. They considered the number of segments for water, the dispersion energy and the association energy during the parameter estimation process. The remaining parameters were left to vary and they were only fitted to vapor pressure and saturated liquid density data. The different parameter sets were used to describe LLE and VLE in binary mixtures of aniline, cyclohexylamine (CHA), hydrocarbons, and water and LLE of some ternary mixtures that contain water. The results of this extensive study showed that good results were obtained from PC-SAFT for predicting LLE of binary water-alkane mixtures, especially with the new parameter set that was published by Grenner et al. ${ }^{16}$. PC-SAFT also performed reasonably well for the binary system water-aniline, but performed poorly for the system water-CHA. For two of the multicomponent systems (wateroctane-aniline, water-CHA-aniline), the experimental data are in good agreement with the results from PC-SAFT, while for the system water-octane-CHA, larger deviations were obtained.

Liang et al. ${ }^{30}$ published an extensive study that focused on pure water properties and phase equilibria of water-containing systems. In their work, they proposed an interactive optimization procedure that estimates the pure component parameters by taking into account LLE data of waterhydrocarbon mixtures in addition to the vapor pressure and saturated liquid density of water. Initially for this process, they fix two of the parameters (association energy and one more) and the other three parameters are fitted to vapor pressure and saturated liquid density data. The association energy was taken gradually from 1660 to $1860 \mathrm{~K}$ with an interval of $20 \mathrm{~K}$. It was found that fixing the association energy and the association volume simultaneously provides the best results. Then, the final parameters were chosen for each association energy based on the deviations of the solubilities of water in the hydrocarbon rich phase. They also compared different parameter sets of both PC-SAFT and CPA on predicting some of pure water's thermodynamic properties including isobaric heat capacity, isochoric heat capacity and speed of sound. Their results showed that it is quite challenging to determine which association scheme is more accurate for water. On one hand, the $2 \mathrm{~B}$ scheme performed better for vapor pressure and isobaric heat capacity, while the 4C scheme performed better for density, isochoric heat capacity and speed of sound. Most notably, both association schemes perform in a similar way, from a qualitative point of view and none of the parameter sets was able to capture the maximum of speed of sound against temperature or the minimum of isochoric heat capacity against temperature in saturated liquid water. At the same 
time, the $2 \mathrm{~B}$ association scheme struggles to accurately describe the mutual solubility of water and hydrocarbon, while the $4 \mathrm{C}$ scheme shows better performance in describing the phase equilibria of these systems. The new parameter set, which was obtained from the new interactive optimization procedure which takes into account LLE data of water-hydrocarbon mixtures, shows accurate description of the phase equilibria of water-hydrocarbon mixtures. At the same time, the new parameter set was quite accurate at predicting the vapor pressure and the density of pure saturated liquid water.

Aparicio-Martinez et al. ${ }^{3}$ have compared different molecular based EOS, like SAFT, PC-SAFT and CPA and they developed new parameter sets for water using different association schemes for each model. They also compared different molecular based EOS, like SAFT, PC-SAFT and CPA. The parameters have been adjusted simultaneously to vapor pressure and saturated liquid density data. They conducted an extensive study where, in addition to these properties, they evaluated the model's performance on predicting the critical point, and the enthalpy and entropy of hydrogen bonding. In their evaluation, they also considered spectroscopic data for monomer fractions in order to examine whether the values of the pure component parameters are in agreement with the experimental values. The published parameter sets are able to predict the vapor pressure and density of saturated liquid water within $8 \%$ and the parameters for all association models give physically realistic results when compared to the enthalpy and entropy of hydrogen bonding. However, the performance of the models seems to decrease at higher temperatures close to the critical temperature. All models also fail to predict the critical point of water. For this purpose, they rescaled the pure-component parameters in order to predict the critical point. The association parameters were not changed in order to maintain their physical meaning. These rescaled parameter sets were indeed able to accurately predict the critical point, but at the cost of the model's performance in describing vapor pressure and density. Finally, they investigated the models' performance in predicting phase equilibria of aqueous binary mixtures that contain carbon dioxide, nitrogen and alkanes. They found that PC-SAFT was superior compared to the other thermodynamic models at describing these properties producing quite satisfactory results.

Diamantonis and Economou ${ }^{37}$ conducted an extensive study by applying PC-SAFT for various compounds that are related to carbon capture applications including water. The pure component parameters were fitted to vapor pressure and saturated liquid density data and they created parameter sets for both $2 \mathrm{~B}$ and $4 \mathrm{C}$ association schemes. They investigated the model's performance for predicting various thermodynamic properties at different conditions. The thermodynamic properties included density, isobaric heat capacity, isochoric heat capacity, isothermal compressibility, speed of sound and Joule-Thomson coefficient. From their study, they concluded that the 4C association scheme is the most suitable for water, since it provided the most accurate results. Their parameter set was able to accurately predict all of these thermodynamic properties within 13\%. The model's accuracy depends on the temperature and pressure conditions of the system. The model's accuracy dropped considerably at conditions close to the critical point.

Yakoumis et al. ${ }^{32}$ applied the CPA equation of state on mixtures that contain hydrocarbons. In their study they published multiple pure-component parameter sets for water with different association schemes. The parameter sets were obtained by simultaneously fitting to vapor pressure and saturated liquid density data. All the parameter sets with different association schemes were able to accurately describe the vapor pressure and the density of saturated liquid water within $2.2 \%$ and there was not any association scheme that performed clearly better compared to the rest. Additionally, they evaluated the performance of different sets on the prediction of mutual solubilities of water-hexane mixtures. Only the parameter set with the $4 \mathrm{C}$ scheme provided 
satisfactory results and for this reason they used this set to predict mutual solubilities of more water-hydrocarbon mixtures. The model provided satisfactory results for these systems considering the estimated uncertainty of the experimental data.

Kontogeorgis et al. ${ }^{38}$ have subsequently applied the CPA equation of state for the prediction of phase equilibria of water-methanol-alkane mixtures and cross-associating water-alcohol mixtures. In this study, they used one of the parameter sets published by Yakoumis et al. ${ }^{32}$. This set used four association sites and they concluded that CPA provided very satisfactory results for all systems both for liquid-liquid equilibria and for vapor-liquid equilibria.

Kontogeorgis et al. ${ }^{39}$ have published a review CPA paper with extensive information about the model and its applications. Pure component parameter sets for many different compounds are presented and CPA's performance is evaluated for predicting pure component properties, including water, and VLE and LLE of some binary mixtures. In this review, there are also parameter sets for water with different association schemes. The pure component parameters of the sets were adjusted to vapor pressure and saturated liquid density data. In this study, there are also guidelines for the pure-component parameter estimation. It is suggested that LLE data can be used to distinguish the different parameter sets. There is also a quite informative discussion about the different association schemes that have been applied for water emphasizing that there is a controversy in the literature about how many sites should be attributed to water. Many years after the study of this publication, it is clear that there is still an ongoing debate about the number of association sites in water. Kontogeorgis et al. ${ }^{39}$ concluded that the CPA equation of state provides quite satisfactory results for the vapor pressure and the density of saturated liquid water. In addition, CPA was able to accurately predict the LLE for water-alkane systems with a single interaction parameter when the $4 \mathrm{C}$ scheme is used for water.

In all the aforementioned studies, different PC-SAFT and CPA parameter sets for water have been presented. In these studies the models' performance has been investigated mostly for saturated liquid density, vapor pressure and phase equilibria, but only few authors (Liang et al. ${ }^{30}$ and Diamantonis et al. ${ }^{37}$ ) have studied other thermodynamic properties as well. Moreover, in none of the manuscripts mentioned above are the models' performance investigated with respect to the prediction of water's anomalies (density maximum, speed of sound maxima, etc.). Water's unique and complex thermodynamic behavior may also be the reason why there are a lot of different parameter sets for different applications and to the fact that there is a continuous debate about the most suitable association scheme.

It should be noted that all of these studies have been published before 2015 and there should be more studies that have published their own pure component parameter sets for water. However, the parameter sets investigated in this work should be enough to properly evaluate the performance of PC-SAFT and CPA on water. It is also worth stating that many recent studies have attempted to modify these models. PC-SAFT and CPA account for hydrogen bonding that is definitely present in water, however there are also more types of interactions in water that are not accounted for in these models. For instance, water is a polar molecule, but in these models the polar interactions are not accounted for. Moreover, it has been reported that water exhibits strong hydrogen-bond cooperativity ${ }^{19,40}$. There have been attempts to incorporate these kind of changes in a molecular EOS. A more detailed discussion about some of these approaches and their ability to predict anomalous behavior is included in Section 5.

\section{Models and database}


4.1. PC-SAFT EOS. Chapman et al. ${ }^{41,42}$ derived the statistical associating fluid theory (SAFT) equation of state based on the Wertheim's thermodynamic perturbation theory ${ }^{5-8}$. Over the past years, many modifications have been suggested for the SAFT model ${ }^{43}$. The PC-SAFT equation of state is a modified version of the original SAFT model and it was developed by Gross and Sadowski ${ }^{43}$ using a new dispersion term based on the perturbation theory of Barker and Henderson $^{44,45}$. The dispersion term in PC-SAFT attempts to account for dispersion forces between whole chains in contrast to the dispersion term of the original SAFT equation, which accounts for dispersion forces between hard spheres ${ }^{43,46}$. The residual Helmholtz free energy for mixtures containing associating fluids in PC-SAFT can be expressed from the following equation ${ }^{29}$ :

$$
a^{r}=a^{h s}+a^{\text {chain }}+a^{\text {disp }}+a^{a s s o c}
$$

where $a^{h s}$ is the Helmholtz free energy contribution of the hard sphere segment-segment interaction, $a^{\text {chain }}$ is the contribution from covalent chain-forming bonds, $a^{\text {disp }}$ is the contribution from the dispersive forces between whole chains and $a^{a s s o c}$ is the contribution from association forces between segments.

In this work, the simplified PC-SAFT model proposed by von Solms et al. ${ }^{47}$ is used. In their work, they propose two main modifications that simplify the expression of the hard sphere term $a^{h s}$ and the expression of the radial distribution function of the hard sphere reference fluid $\mathrm{g}^{h s}$. It is worth noting that for pure compounds the results of original PC-SAFT and of the simplified PCSAFT are identical. The hard sphere term in the simplified version is expressed as:

$$
\frac{a^{h s}}{R T}=\frac{4 \eta-3 \eta^{2}}{(1-\eta)^{2}}
$$

where $\eta$ is the packing fraction of the mixture. The chain term $a^{\text {chain }}$ can be expressed as:

$$
\frac{a^{\text {chain }}}{R T}=\sum_{i} x_{i}\left(1-m_{i}\right) \ln \left(g_{i i}\left(d_{i i}\right)^{h s}\right)
$$

where $R$ is the gas constant, $T$ the temperature of the system, $x_{i}$ the mole fraction of molecule $i$, $m_{i}$ the chain length of molecule $i, g_{i i}$ the radial pair distribution function for segments of component $i$ and $d_{i i}$ the temperature dependent diameter.

The dispersion term $a^{\text {disp }}$ can be expressed:

$$
\frac{a^{d i s p}}{k T N}=\frac{a_{1}}{k T N}+\frac{a_{2}}{k T N}
$$

Where $k$ is the Boltzmann constant, $a_{1}$ is the first-order term and $a_{2}$ is the second-order term. These terms can be expressed as:

$$
\frac{a_{1}}{k T N}=-2 \pi \rho m^{2}\left(\frac{\varepsilon}{k T}\right) \sigma^{3} \int_{1}^{\infty} \tilde{u}(x) g^{h c}(m ; x \sigma / d) x^{2} d x
$$




$$
\begin{gathered}
\frac{a_{2}}{k T N}=-\pi \rho m\left(1+Z^{h c}\right. \\
\left.+\rho \frac{\partial Z^{h c}}{\partial \rho}\right)^{-1} m^{2}\left(\frac{\varepsilon}{k T}\right)^{2} \sigma^{3} \frac{\partial}{\partial \rho}\left[\rho \int_{1}^{\infty} \tilde{u}(x)^{2} g^{h c}(m ; x \sigma / d) x^{2} d x\right]
\end{gathered}
$$

where $\varepsilon$ is the segment energy parameter, $x$ is the reduced radial distance around a segment, $\tilde{u}(x)$ is the reduced intermolecular potential, $g^{h c}(m ; x \sigma / d)$ is the average segment-segment radial distribution function of the hard-chain fluid with temperature dependent segment diameter $d$ and $Z^{h c}$ is the hard chain contribution to the compressibility factor $Z$.

The association term $a^{a s s o c}$ can be expressed as:

$$
\frac{a^{a s s o c}}{R T}=\sum_{i} x_{i}\left[\sum_{A_{i}}\left(\ln X^{A_{i}}-\frac{X^{A_{i}}}{2}\right)+\frac{1}{2} M_{i}\right]
$$

where $X^{A_{i}}$ is the fraction of sites A on molecule $i$ not bonded to other active sites and $M_{i}$ is the number of association sites on molecule $i$. The second sum in equation (7) represents a sum over all association sites of the molecule. The monomer fraction $X^{A_{i}}$ can be obtained from:

$$
X^{A_{i}}=\left[1+\sum_{j} \sum_{B_{j}} \rho_{j} X^{B_{j}} \Delta^{A_{i} B_{j}}\right]^{-1}
$$

where $\rho_{j}$ is the molar density of molecule $j$ and $\Delta^{A_{i} B_{j}}$ is the association strength between two sites $A$ and $B$ belonging to two different molecules $i$ and $j$.

For the application of this equation of state five pure-component parameters need to be specified for each associating fluid (or three for non-associating fluids). These parameters have physical meaning and they are:

(1) $m$ : chain length or segment number

(2) $\sigma$ : temperature independent segment diameter

(3) $\varepsilon$ : segment energy parameter

(4) $\kappa^{A_{i} B_{i}}$ : association volume

(5) $\varepsilon^{A_{i} B_{i}}$ : association energy

These parameters are typically fitted simultaneously to vapor pressure and saturated liquid density data. More extensive information on the PC-SAFT EOS is available in the original literature ${ }^{34,43}$. More information on the modifications is available in the work of von Solms et al. ${ }^{47}$.

4.2. CPA EOS. The cubic-plus-association (CPA) equation of state, developed by Kontogeorgis et al. ${ }^{48}$, combines the classical Soave-Redlich-Kwong (SRK) equation with an association term similar to the association term of the SAFT EOS. For non-associating fluids the CPA EOS reduces to SRK. Thus, this model achieves a balance between accuracy due to the inclusion of the association term and simplicity as all physical interactions are represented by $\mathrm{SRK}^{46}$. The CPA can be expressed in terms of residual Helmholtz free energy $\mathrm{as}^{49}$ : 


$$
a^{r}=a^{S R K}+a^{a s s o c}
$$

where $a^{S R K}$ is the Helmholtz free energy contribution of the SRK equation of state, which contains the contribution of repulsive and dispersive interactions and $a^{a s s o c}$ is the contribution of association. These terms can be expressed as:

$$
\begin{aligned}
& \frac{a^{S R K}}{n R T}=-\ln \left(1-\frac{b}{v}\right)-\frac{a(T)}{b R T} \ln \left(1+\frac{b}{v}\right) \\
& \frac{a^{a s s o c}}{R T}=\sum_{i} n_{i} \sum_{A_{i}}\left(\ln X^{A_{i}}-\frac{1}{2} X^{A_{i}}+\frac{1}{2}\right)
\end{aligned}
$$

where $n$ is the total number of moles, $b$ is the co-volume parameter, $v$ is the molar volume, $a(T)$ is the temperature dependent energy parameter of the mixture and $X^{A_{i}}$ is the monomer fraction (equation 7). It is important to note that the association strength $\Delta^{A_{i} B_{j}}$ has the same physical meaning in both equations, but they have slightly different expressions.

For the application of this equation of state five pure-component parameters need to be specified for each associating fluid (or three for non-associating fluids). These parameters are:

(1) $b$ : co-volume parameter

(2) $a_{0}$ : constant parameter in the temperature dependent energy parameter $a(T)$

(3) $c_{1}$ : constant parameter in the temperature dependent energy parameter $a(T)$

(4) $\varepsilon^{A_{i} B_{i}}$ : association energy parameter

(5) $\beta^{A_{i} B_{i}}$ : association volume parameter

These parameters are typically adjusted simultaneously to vapor pressure and saturated liquid density data. More information on the CPA equation of state is available from Kontogeorgis et al. $^{46}$

4.3. Derived properties. In this work, some thermodynamic properties are examined, including residual isobaric heat capacity, the residual isochoric heat capacity and the speed of sound which are connected to the second order derivatives of the residual Helmholtz energy. For the calculation of the speed of sound the ideal isobaric heat capacity is required, which is calculated from the equation available in the DIPPR database ${ }^{29}$.

$$
\begin{gathered}
C_{V}^{r}=-T\left(\frac{\partial^{2} a^{r}}{\partial T^{2}}\right)_{V, n} \\
C_{P}^{r}=-T\left(\frac{\partial^{2} a^{r}}{\partial T^{2}}\right)_{V, n}+T\left(\frac{\partial P}{\partial T}\right)_{V, n}\left(\frac{\partial V}{\partial T}\right)_{V, n}-R
\end{gathered}
$$




$$
u=\sqrt{-\frac{V^{2}}{M_{w}} \frac{C_{P}}{C_{V}}\left(\frac{\partial P}{\partial V}\right)_{V, n}}
$$

where $M_{w}$ is the molecular weight.

In order to evaluate the performance of the models, the percentage average absolute relative deviation \% AAD is used which can be expressed as:

$$
\% A A D(\Omega)=\frac{1}{N_{p}} \sum_{i=1}^{N_{p}}\left|\frac{\Omega_{i}^{\text {calc }}}{\Omega_{i}^{\text {exp }}}-1\right| \cdot 100 \%
$$

where $\Omega$ is the vapor pressure, density, residual isobaric heat capacity, residual isochoric heat capacity or speed of sound and $N_{p}$ is the number of (experimental and calculated) points.

4.4. Database. For evaluating the performance of the equations of state in describing water's thermodynamic properties, a large collection of experimental data is used that covers a wide range of temperatures and pressures. In some cases, the experimental data were insufficient and so the predicted values from the advanced EOS are also compared with the results from the NIST model $^{21}$. The values from the NIST model ${ }^{21}$ are extremely close to the experimental ones and thus these values can be used as alternative reliable sources when there is a lack of experimental data. In fact, other authors ${ }^{3,30,37,38}$ have studied the performance of the PC-SAFT and CPA equations of state on modeling water by comparing the predicted values with results from the NIST model ${ }^{21}$ or the IAPWS-95 formulation ${ }^{22}$. Table 1 shows the ranges of the data and the NIST model results that were used for the evaluation of the models. For most properties, results from the NIST model ${ }^{21}$ were used in order to cover also the high temperatures (up to 640-645 K). For the isochoric heat capacity $C_{V}^{r}$ only results from the NIST model ${ }^{21}$ were used.

Table 1. Overview of experimental data and results from the NIST model that were used for evaluating the performance of PC-SAFT and CPA ${ }^{\mathrm{a}}$.

\begin{tabular}{|c|c|c|c|c|c|}
\hline \multirow{2}{*}{ Property } & \multicolumn{2}{|c|}{ Range } & \multicolumn{2}{c|}{ No. of points } & \multirow{2}{*}{ Sources } \\
\cline { 2 - 5 } & T (K) & P (MPa) & Exp. & NIST & \multirow{2}{*}{${ }^{23-26}$, NIST $^{21}$} \\
\hline$\rho$ & $273-640$ & $0.1-800$ & 280 & 78 & Data $^{22}$ \\
\hline$C_{P}^{r}$ & $273-640$ & $0.1-400$ & 68 & 203 & Data $^{28}$, NIST $^{21}$ \\
\hline$C_{V}^{r}$ & $275-640$ & $0.1-400$ & - & 269 & NIST $^{21}$ \\
\hline$u$ & $273-640$ & $0.1-600$ & 299 & 362 & Data $^{50-52}$, NIST $^{21}$ \\
\hline$P^{S}$ & $273-646$ & - & 444 & - & Data $^{23,53-57}$ \\
\hline$\rho$ (Saturated) & $273-645$ & - & 91 & - & Data $^{23,24,58,59}$ \\
\hline$C_{P}^{r}$ (Saturated) & $283-630$ & - & 36 & - & Data $^{60}$ \\
\hline$C_{V}^{r}$ (Saturated) & $275-630$ & - & - & 72 & NIST $^{21}$ \\
\hline$u$ (Saturated) & $273-645$ & - & 108 & 26 & Data $^{61}$, NIST $^{21}$ \\
\hline
\end{tabular}


aNotes: (1) The residual isobaric heat capacity and residual isochoric heat capacity values were calculated by using ideal gas isobaric heat capacity and ideal gas isochoric heat capacity values from the DIPPR database ${ }^{29}$. (2) The maximum uncertainty (of all the experimental data of all the properties) is estimated to be $\pm 0.5 \%$.

\section{Results and Discussion}

5.1 Quantitative performance of the models Twelve PC-SAFT parameter sets and two CPA parameter sets for pure water are used and compared for the prediction of pure liquid water's thermodynamic properties. The parameter sets were previously published in the studies mentioned in section 3. The parameter sets for PC-SAFT and for CPA are listed in Table 2 and Table 3 respectively. Names have been assigned to the different parameter sets in order to classify them. The first letter in the names refers to the model ("P" for PC-SAFT and "C" for CPA) then followed by the association scheme used $(2 \mathrm{~B}, 3 \mathrm{~B}$ or $4 \mathrm{C})$.

Table 2. Water pure component parameters for PC-SAFT.

\begin{tabular}{|c|c|c|c|c|c|c|c|}
\hline Sets & $m$ & $\sigma(\AA)$ & $\varepsilon / k(\mathrm{~K})$ & $\varepsilon^{A_{i} B_{i}} / k(\mathrm{~K})$ & $\kappa^{A_{i} B_{i}}$ & Scheme & Ref. \\
\hline P2B_1 & 1.0656 & 3.0007 & 366.51 & 2500.07 & 0.034868 & 2B & 34 \\
\hline P2B_2 & 1.3112 & 2.7613 & 372.37 & 2123.10 & 0.048987 & 2B & 3 \\
\hline P2B_3 & 1.9599 & 2.362 & 279.42 & 2059.28 & 0.1750 & 2B & 37 \\
\hline P2B_4 & 1.09528 & 2.8898 & 365.956 & 2515.6706 & 0.034868 & 2B & 35 \\
\hline P3B_1 & 1.7960 & 2.4697 & 327.62 & 1558.40 & 0.068277 & 3B & 3 \\
\hline P3B_2 & 2.3753 & 2.5609 & 275.81 & 1558.40 & 0.068277 & 3B & 3 \\
\hline P4C_1 & 1.5 & 2.6273 & 180.30 & 1804.22 & 0.0942 & 4C & 16 \\
\hline P4C_2 & 2.1945 & 2.2290 & 141.66 & 1804.17 & 0.2039 & 4C & 37 \\
\hline P4C_3 & 3.0 & 2.0135 & 182.92 & 1259.00 & 0.4287 & 4C & 15 \\
\hline P4C_4 & 1.5725 & 2.6270 & 291.13 & 1334.20 & 0.074347 & 4C & 3 \\
\hline P4C_5 & 2.0 & 2.3449 & 171.67 & 1704.06 & 0.1596 & 4C & 30 \\
\hline P4C_6 & 2.7500 & 2.0794 & 183.61 & 1354.1 & 0.3374 & 4C & 15 \\
\hline
\end{tabular}

Table 3. Water pure component parameter sets for $\mathrm{CPA}^{\mathrm{b}}$.

\begin{tabular}{|c|c|c|c|c|c|c|c|}
\hline Sets & $\begin{array}{c}b \\
(\mathrm{~L} / \mathrm{mol})\end{array}$ & $\begin{array}{c}\Gamma \\
(K)\end{array}$ & $c_{1}$ & $\begin{array}{c}\varepsilon^{A_{i} B_{i}} \\
(K)\end{array}$ & $\begin{array}{c}\beta^{A_{i} B_{i}} \\
\left(10^{3}\right)\end{array}$ & Scheme & Ref. \\
\hline C3B_1 & 0.014969 & 2415.2174 & 0.35928 & 2501.3042 & 21.3 & $3 \mathrm{~B}$ & 39 \\
\hline C4C_1 & 0.0145 & 1018.3336 & 0.6736 & 2003.1361 & 69.2 & $4 \mathrm{C}$ & 32,38 \\
\hline
\end{tabular}

bote: (1) The expression of $\Gamma$ is $\Gamma=a_{0} /(R \cdot b)$

Table 4 shows an overview of the parameter sets used along with information on the properties used in the fitting of the water model parameters. In most cases, the pure component parameters have been adjusted only to vapor pressure and saturated liquid density data. There are a few parameter sets that have used different approaches to assign values to the parameters. The set P3B_2 from Aparicio-Martinez ${ }^{3}$ is based on the rescaling of the parameters of set P3B_1 in order 
to accurately predict the critical point of water. In the rescaling process, the association parameters were not readjusted and for this reason the sets P3B_1 and P3B_2 have the same values for the association parameters (Table 2). The set P4C_1 published by Grenner et al. ${ }^{16}$ has some of the parameters fixed according to their physical meaning. Similarly, the sets P4C_3 and P4C_6 from von Solms et al. ${ }^{15}$ have a fixed parameter. Among the parameter sets discussed in this study, only the sets P4C_5, C3B_1, C4C_1 have parameters that have been selected based on their performance on predicting phase equilibria of aqueous systems.

Tables 5 and 6 show the absolute average deviation (\%AAD) between the calculated with PCSAFT and CPA and the experimental (or NIST) values using the various parameter sets. Table 5 shows \%AAD values for saturated conditions and table 6 for unsaturated conditions. The reported $\%$ AAD values for vapor pressure $P^{S}$ and saturated liquid density $\rho^{S}$ in Table 4 can have differences from the \%AAD values calculated in this work. This is mostly due to the fact of the different temperature ranges used in Table 4 (literature) and Tables 5-6 (this work).

Table 4. Information about the pure component parameter sets. ${ }^{c}$

\begin{tabular}{|c|c|c|c|c|c|}
\hline \multirow[t]{2}{*}{ Sets } & \multirow[t]{2}{*}{ Authors } & \multicolumn{2}{|c|}{$\begin{array}{l}\text { Reported } \\
\% A A D\end{array}$} & \multirow{2}{*}{$\begin{array}{l}\text { T Range } \\
\text { (K) }\end{array}$} & \multirow{2}{*}{$\begin{array}{c}\text { Data used in parameter } \\
\text { estimation }\end{array}$} \\
\hline & & $P^{S}$ & $\rho^{S}$ & & \\
\hline P2B_1 & $\begin{array}{c}\text { Gross and } \\
\text { Sadowski }\end{array}$ & 1.88 & 6.83 & $273-647$ & $P^{S}, \rho^{S}$ \\
\hline P2B_2 & $\begin{array}{l}\text { Aparicio-Martínez, } \\
\text { and Hall }\end{array}$ & 1.03 & 6.67 & $273-634$ & $P^{S}, \rho^{S}$, spectroscopy \\
\hline P2B_3 & $\begin{array}{l}\text { Diamantonis and } \\
\text { Economou }^{37}\end{array}$ & 1.18 & 3.92 & $275-640$ & $P^{S}, \rho^{S}$ \\
\hline P2B_4 & Cameretti et al. ${ }^{35}$ & $<0.9$ & $<0.9$ & $278-393$ & $P^{S}, \rho^{S}$ \\
\hline P3B_1 & $\begin{array}{l}\text { Aparicio Martinez } \\
\text { and } \mathrm{Hall}^{3}\end{array}$ & 0.87 & 6.36 & $273-634$ & $P^{S}, \rho^{S}$, spectroscopy \\
\hline P3B_2 & $\begin{array}{l}\text { Aparicio Martinez } \\
\text { and } \mathrm{Hall}^{3} \\
\end{array}$ & 4.35 & 61.1 & $273-634$ & $\begin{array}{l}P_{C}, T_{C}(\text { rescaled P3B_1 }), \text { fixed } \\
\varepsilon^{H B}, \text { fixed } \kappa^{H B}, \text { spectroscopy }\end{array}$ \\
\hline P4C_1 & Grenner et al. ${ }^{16}$ & 0.93 & 2.62 & $324-582$ & $\begin{array}{c}P^{S}, \rho^{S}, \text { fixed } m, \text { fixed } \varepsilon, \text { fixed } \\
\varepsilon^{H B}\end{array}$ \\
\hline P4C_2 & $\begin{array}{c}\text { Diamantonis and } \\
\text { Economou }^{37}\end{array}$ & 1.98 & 0.83 & $275-640$ & $P^{S}, \rho^{S}$ \\
\hline P4C_3 & von Solms et al. ${ }^{15}$ & 0.43 & 1.12 & $324-582$ & $P^{S}, \rho^{S}$, fixed $m$, spectroscopy \\
\hline P4C_4 & $\begin{array}{l}\text { Aparicio Martinez } \\
\text { and Hall } \\
\end{array}$ & 1.00 & 7.66 & $273-634$ & $P^{S}, \rho^{S}$, spectroscopy \\
\hline P4C_5 & Liang et al. ${ }^{30}$ & 1.46 & 2.14 & $280-620$ & $\begin{array}{c}P^{S}, \rho^{S}, \text { fixed } \varepsilon^{H B}, \text { fixed } \kappa^{H B}, \\
\text { phase equilibria of water- } \\
\text { hydrocarbons }\end{array}$ \\
\hline P4C_6 & von Solms et al. ${ }^{15}$ & 0.44 & 1.05 & $324-582$ & $P^{S}, \rho^{S}$, fixed $m$, spectroscopy \\
\hline C3B_1 & Kontogeorgis et al. ${ }^{39}$ & 0.37 & 0.17 & $356-582$ & $\begin{array}{c}P^{S}, \rho^{S}, \text { phase equilibria of water } \\
\text { hydrocarbons }\end{array}$ \\
\hline
\end{tabular}




\begin{tabular}{|c|c|c|c|c|c|}
\hline C4C_1 & Yakoumis et al. $^{32}$ & 0.77 & 0.33 & $278-582$ & $\begin{array}{c}P^{S}, \rho^{S}, \text { phase equilibria of } \\
\text { water-hydorcarbons }\end{array}$ \\
\hline
\end{tabular}

'Notes: (1) The \%AAD values and T ranges in the table are the ones reported by the authors. (2) Cameretti et al. ${ }^{35}$ did not publish exact \%AAD values for their parameter set. They also plotted the calculated values against the experimental ones in order to show the performance of the parameter set. (3) $P^{S}$ is the vapor pressure, $\rho^{S}$ is the density under saturated conditions, $P_{C}$ is the critical pressure and $T_{C}$ is the critical temperature, $m$ is the chain length, $\varepsilon$ is the segment energy parameter, $\varepsilon^{H B}$ is the association energy parameter, $\kappa^{H B}$ is the association volume. (4) The data from spectroscopy and from phase equilibria in all these cases were not used as part of the objective function to fit the parameters, but they were used to either fix specific parameters to values or to choose the final values of the parameter (see also Section 3).

Table 5. \%AAD for pure liquid water's properties in saturated conditions ${ }^{\mathrm{d}}$.

\begin{tabular}{|c|c|c|c|c|c|c|}
\hline \multirow{2}{*}{ Set } & \multicolumn{6}{|c|}{ \%AAD } \\
\cline { 2 - 7 } & $P^{S}$ & $\rho$ & $C_{P}^{r}$ & $C_{V}^{r}$ & $u$ & Sum \\
\hline P2B_1 & 1.09 & 11.2 & 26.3 & 22.8 & 48.5 & 110.0 \\
\hline P2B_2 & 1.37 & 10.8 & 15.7 & 20.1 & 63.1 & 111.2 \\
\hline P2B_3 & 1.37 & 7.48 & 11.3 & 26.4 & 53.4 & 99.9 \\
\hline P2B_4 & 5.15 & 12.0 & 25.2 & 22.3 & 50.0 & 114.7 \\
\hline P3B_1 & 1.10 & 10.4 & 15.4 & 29.4 & 75.1 & 131.3 \\
\hline P3B_2 & 3.07 & 38.1 & 27.5 & 19.1 & 65.6 & 153.5 \\
\hline P4C_1 & $\mathbf{0 . 8 8}$ & 10.4 & 30.4 & 20.2 & 23.7 & 85.7 \\
\hline P4C_2 & 1.67 & $\mathbf{4 . 2 8}$ & 20.6 & 23.9 & 12.3 & 62.8 \\
\hline P4C_3 & 1.03 & 6.67 & $\mathbf{1 0 . 8}$ & 34.7 & 53.3 & 106.6 \\
\hline P4C_4 & 1.45 & 12.5 & 15.5 & 19.3 & 69.8 & 118.4 \\
\hline P4C_5 & 1.08 & 7.46 & 21.5 & 22.0 & 26.7 & 78.8 \\
\hline P4C_6 & 1.00 & 7.09 & 13.0 & 31.4 & 48.5 & 101.0 \\
\hline C4C_1 & 1.08 & 5.40 & 12.1 & $\mathbf{1 5 . 0}$ & $\mathbf{1 1 . 6}$ & $\mathbf{4 5 . 2}$ \\
\hline C3B_1 & 1.91 & 9.16 & 30.1 & 34.3 & 34.4 & 109.8 \\
\hline
\end{tabular}

dNotes: (1) The sum in the final column refers to the sum of all the \%AAD values for a particular set. (2) The bold \%AAD values indicate the lowest per column i.e. per property among all sets and both models (3) The maximum estimated uncertainty of the experimental data for these properties is $\pm 0.5 \%$. Thus, none of the sets provides results within the uncertainty of the measurements.

From Table 5 it can be concluded that most parameter sets are able to accurately predict the vapor pressure $P^{S}$ within $2 \%$. This is not surprising since pure component parameters are usually fitted to vapor pressure and saturated liquid density data. There are two parameter sets (P3B_2 and P2B_4) that show a slightly higher deviation compared to the other sets. The parameters of the set $\mathrm{P} 3 \mathrm{~B} \_2$ published by Aparicio-Martinez ${ }^{3}$ were fitted to the critical point of water. Therefore, this set is able to accurately predict the critical point of water, at the cost of other thermodynamic properties, including the crucial vapor pressure. In the set P2B_4 from Cameretti et $\mathrm{al}^{35}$, the parameters were adjusted at the $T$ range $278-393 \mathrm{~K}$ (Table 4 ). This range is much 
narrower than the range used in our work and with all models, which explains the difference between the reported \%AAD and the \%AAD values of this work.

The most accurate set at predicting the vapor pressure seems to be $\mathrm{P} 4 \mathrm{C} \_1$ with an accuracy of $0.88 \%$. This is a bit surprising considering the fact that the parameters were adjusted at the T range 324-582. For this set, some parameters (Table 4) were fixed to values acquired from spectroscopy data and molecular simulations ${ }^{16}$. The maximum uncertainty of the vapor pressure data is estimated to be $\pm 0.04 \%$. Thus, even the most accurate set is not within the experimental uncertainty and that is partly because of the extremely accurate experimental data.

Table 6. \%AAD for pure liquid water's properties in unsaturated conditions ${ }^{\mathrm{e}}$.

\begin{tabular}{|c|c|c|c|c|c|}
\hline \multirow{2}{*}{ Sets } & \multicolumn{5}{|c|}{ \%AAD } \\
\cline { 2 - 6 } & $\rho$ & $C_{P}^{r}$ & $C_{V}^{r}$ & $u$ & Sum \\
\hline P2B_1 & 9.59 & 9.29 & 20.3 & 38.1 & 77.3 \\
\hline P2B_2 & 7.19 & 6.42 & 13.2 & 47.5 & 74.3 \\
\hline P2B_3 & 5.81 & $\mathbf{2 . 5 8}$ & 17.7 & 41.5 & 67.6 \\
\hline P2B_4 & 2.72 & 9.44 & 20.2 & 37.5 & 69.9 \\
\hline P3B_1 & 7.29 & 6.74 & 21.1 & 55.6 & 90.7 \\
\hline P3B_2 & 38.3 & 24.8 & $\mathbf{7 . 8 9}$ & 61.9 & 132.9 \\
\hline P4C_1 & 3.90 & 20.5 & 17.4 & 16.9 & 58.7 \\
\hline P4C_2 & $\mathbf{0 . 7 6}$ & 14.0 & 22.1 & $\mathbf{9 . 6 7}$ & 46.6 \\
\hline P4C_3 & 3.21 & 5.93 & 27.8 & 39.5 & 76.5 \\
\hline P4C_4 & 7.80 & 4.62 & 10.6 & 49.9 & 72.9 \\
\hline P4C_5 & 3.05 & 12.5 & 18.2 & 19.8 & 53.5 \\
\hline P4C_6 & 2.98 & 6.57 & 24.8 & 35.8 & 70.1 \\
\hline C4C_1 & 1.62 & 4.10 & 13.4 & 17.2 & $\mathbf{3 6 . 4}$ \\
\hline C3B_1 & 2.58 & 18.3 & 34.5 & 39.4 & 94.8 \\
\hline
\end{tabular}

eNotes: (1) The sum in the final column refers to the sum of all the \%AAD values for a particular set. (2) The bold \%AAD values indicate the lowest per column i.e. per property among all sets and both models (3) The maximum estimated uncertainty of the experimental data for these properties is $\pm 0.5 \%$. Thus, none of the sets provides results within the uncertainty of the measurements.

From Tables 5 and 6 it can be concluded that most of the parameter sets are able to represent the liquid density within 13\%. The set P3B_2 cannot predict accurately this property (error of $38.2 \%$ ), which is maybe to be expected since this property was not used in the parameter estimation. The most accurate set for predicting the density of liquid water is P4C_2 and for both saturated (accuracy of $4.28 \%$ ) and unsaturated conditions (accuracy of $0.76 \%$ ). Even though the parameter sets were not fitted to unsaturated liquid density data, both models are in general more accurate at predicting density in unsaturated conditions rather than in saturated conditions. For instance, the set $\mathrm{C} 3 \mathrm{~B} \_1$ is able to predict saturated liquid density within $9.16 \%$, but in unsaturated conditions it is able to predict density values within $2.58 \%$.

The other thermodynamic properties are not predicted as accurately as density and it is likely related to the fact that none of the parameter sets have been obtained using these other properties 
in the parameter estimation. For instance, the set P4C_2, which was the most accurate for density in unsaturated conditions $(0.76 \%)$ showed an error of more than $9 \%$ for the rest of the thermodynamic properties. Of course there are also occasions where a parameter set could be more accurate at predicting a second-order derivative property than density, like the set P4C_4. The set P4C_4 is able to predict $C_{P}^{r}$ in unsaturated conditions within $4.62 \%$, but the set's accuracy at predicting density values is $7.80 \%$. This is quite interesting considering the fact that this set was not fitted to heat capacity data but they were fitted to density data. The reason why some sets are more accurate at the other thermodynamic properties seems unclear and a more extensive analysis would be required in order to properly explain this phenomenon.

The set P4C_3 is the most suitable set for predicting the $C_{P}^{r}$ of saturated liquid water according to Table 5 with an accuracy of $10.8 \%$, but for unsaturated liquid water the set P2B_3 is the most accurate in predicting $C_{P}^{r}$ values $(2.58 \%)$. So even if a particular set is the most accurate at predicting a property of saturated liquid water, it is possible that the same parameter set is not the most accurate at predicting the same property in unsaturated liquid water over the investigated temperature and pressure ranges. This can also be observed for the other properties. The set C4C_1 is the most accurate at predicting the $C_{V}^{r}(15.0 \%)$ and the speed of sound of saturated liquid water (11.6\%), but the set P3B_2 is the most suitable one for accurately predicting $C_{V}^{r}$ of unsaturated liquid water $(7.89 \%)$ and the set P4C_2 provides the most accurate prediction of the speed of sound of unsaturated liquid water $(9.67 \%)$. It is quite interesting that the set P3B_2, which is the set that was based on critical point of water, shows satisfactory results also for $C_{V}^{r}$. The set P4C_2 shows the best results for density in addition to the speed of sound at unsaturated conditions.

The parameter set that shows the least sum of errors for all properties is the C4C_1 (sum of errors is $36.4 \%$ in unsaturated conditions and $45.2 \%$ in saturated conditions), which is the CPA parameter set for water using the $4 \mathrm{C}$ i.e. the parameter set typically used in CPA calculations for aqueous systems. In other words, this set has been reported to show exceptional results for the prediction of phase equilibria of aqueous systems. However, the fact that it has the least sum of errors at predicting pure liquid water properties along with great accuracy of predicting LLE data of various aqueous mixtures does not indicate that it should automatically be preferred over the other sets. There are a lot of factors to consider and also the fact that this set was able to perform better from a quantitative point of view does not naturally indicate that the set also provides qualitatively correct results.

Overall, Tables 5 and 6 provide important information on the accuracy of the models for the different parameter sets. From these tables it is also possible to conclude which of these parameter sets is most accurate at describing a specific property. However, it should be noted that none of the sets is able to provide results within experimental uncertainty and this is partly due to the fact that the data are extremely accurate (maximum estimated uncertainty of $\pm 0.5 \%$ ). It is also clear from Tables 5 and 6 that the models tend to be more accurate in unsaturated conditions than in saturated conditions. Figures 5-11 show that the performance of the models depends heavily on pressure. For instance, the set P4C_2 shows exceptional accuracy at pressures 100-200 MPa (Figure 14), but at $0.1 \mathrm{MPa}$, where there is a maximum in the T-dependence of $\rho$, the set is not as accurate. 


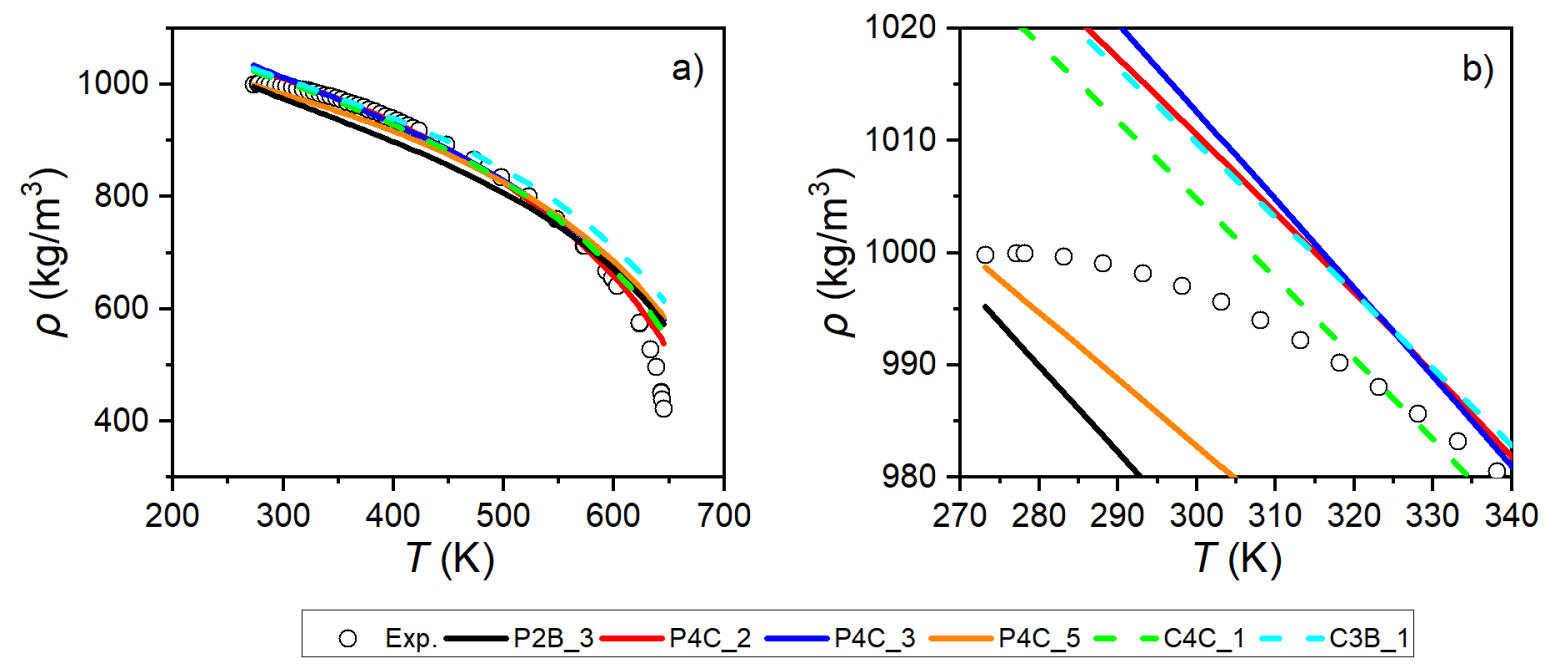

Figure 5. Experimental and calculated density of pure saturated liquid water. a) Plot of all the experimental and calculated values b) Enlarged graph to emphasize the maximum. The symbols represent the experimental data ${ }^{23,24,58,59}$ (Table 1) and the lines the calculated values with PC-SAFT and CPA with different parameter sets.

5.2. Qualitative performance of the models and prediction of extrema. Tables 5 and 6 can provide detailed information on the model's performance for the various properties. However, examining only the \%AAD values is not always enough in order to evaluate the model's qualitative (and quantitative) capabilities in predicting the anomalous thermodynamic behavior of water. It is important to investigate whether the models are able to predict the various extrema, like those shown in figures 3 and 4.

Figure 5 shows the experimental and calculated values of the saturated liquid density. There is actually a maximum at approximately $277 \mathrm{~K}$. From Figure $5 \mathrm{~b}$ it is clear that none of the models is able to predict this maximum. This figure also shows that all models have qualitatively the same behavior, irrespectively of different quantitative results. In addition, at temperatures close to the critical point of water $(647 \mathrm{~K})$, the actual density values significantly decrease with respect to temperature. This behavior is also not predicted by the models and it seems that the model's performance at predicting this property significantly drops at temperatures close to the critical point. This decrease in accuracy is not surprising. At temperatures close to the critical point of a pure substance, intense density fluctuations are occurred. These density fluctuations are not taken into account by CPA and PC-SAFT.

Figure 6 shows the experimental and calculated residual isobaric heat capacity of saturated liquid water. This property displays a minimum at low temperatures. As shown in Figure 6, none of the models is able to predict the curvature of the residual isobaric heat capacity values against temperature including the minimum. Out of these six parameter sets, only the set P4C_3 predicts a minimum, but at much higher temperature $(\approx 400 \mathrm{~K})$ compared to the actual one $(\approx 330 \mathrm{~K})$. In addition the models show qualitatively different results for the calculated $C_{P}^{r}$ values, unlike the calculated density values. At temperatures close to the critical point, the model's accuracy at predicting this property significantly decreases, similarly to the prediction of density.

Figure 7 shows the models' qualitative performance for the residual isochoric heat capacity and the speed of sound of saturated liquid water. The actual isochoric heat capacity of saturated 
liquid water displays a minimum at high temperature, but none of the models can show this. The sets C4C_1 and C3B_1 actually display maxima in Figure 7a. Finally, as shown in Figure 7b, all of the calculated models and sets fail to accurately describe the behavior of the speed of sound with respect to temperature. The experimental speed of sound values show a maximum, but all of the calculated values continuously increase when the temperature decreases.

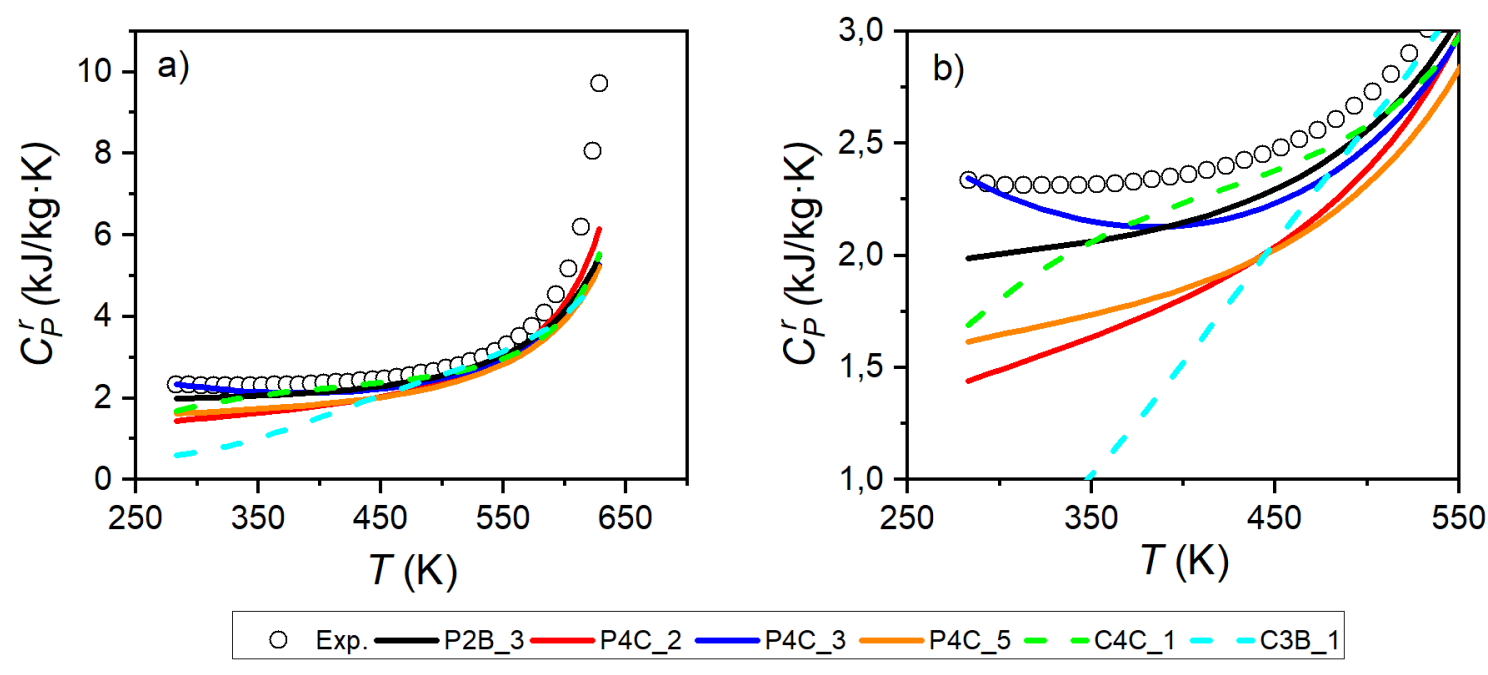

Figure 6. Experimental and calculated residual isobaric heat capacity of saturated liquid water. a) Plot of all the experimental and calculated values b) Enlarged graph to emphasize the minimum. The symbols represent the experimental data ${ }^{60}$ (Table 1) and the lines represent the calculated values from PC-SAFT and CPA with different parameter sets.

Figure 8 shows experimental and calculated density isobars of pure water at four different pressures. At $0.1 \mathrm{MPa}$ all models and parameter sets fail to predict the curvature shown by the data. The experimental values show a maximum, while the calculated densities continuously increase when the temperature decreases. At the higher pressures (100 MPa-400MPa), the models are able to predict the curvature of the density isobars, possibly because there is no longer a density maximum. It is clear that the models have difficulties in accurately predicting water's properties, because of water's anomalous thermodynamic behavior. 


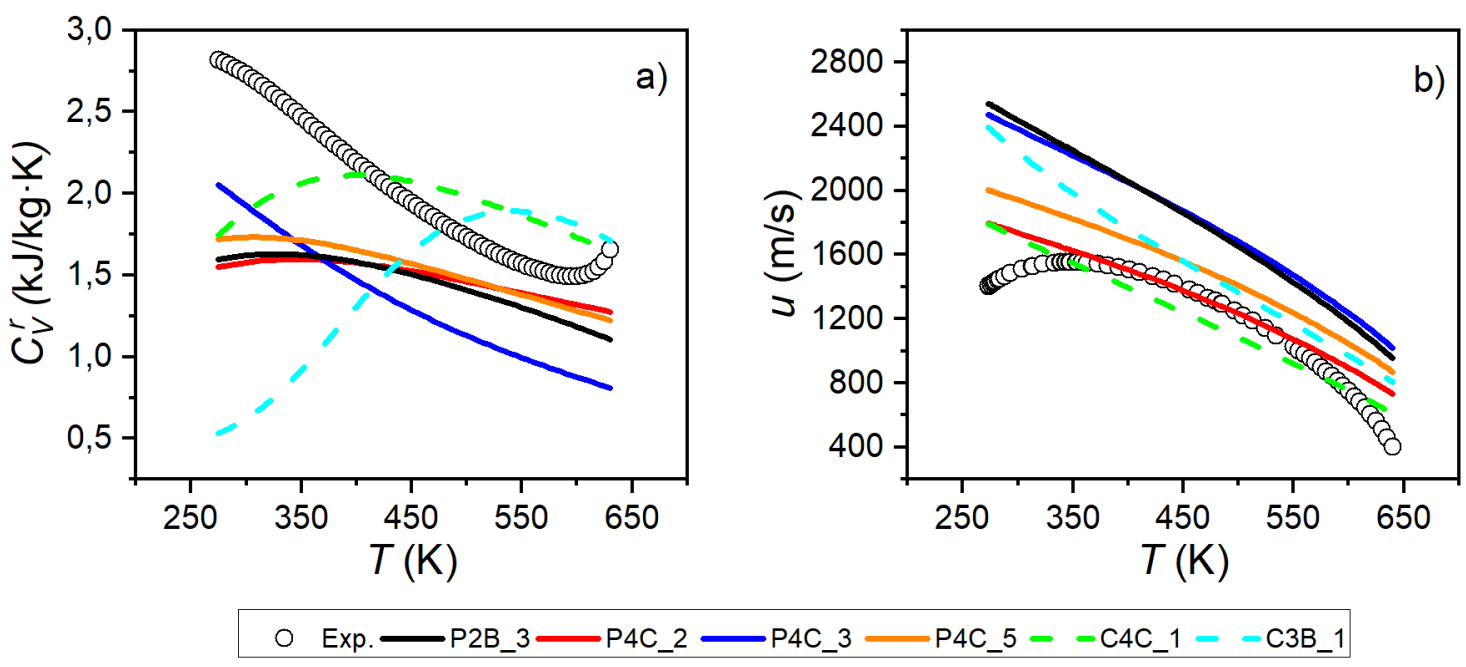

Figure 7. Experimental and calculated thermodynamic properties of pure saturated liquid water with PC-SAFT and CPA with different parameter sets. a) Residual isochoric heat capacity. The symbols represent the results from the NIST model ${ }^{21}$ and the lines the calculated values from PCSAFT and CPA b) Speed of sound. The symbols represent experimental data ${ }^{61}$ and results from the NIST model ${ }^{21}$ and the lines the calculated values from PC-SAFT and CPA.

Figure 9 shows experimental and calculated $C_{P}^{r}$ isobars of pure liquid water at four different pressures. The temperature and pressure dependence of this property is quite complex. There is a $C_{P}^{r}$ minimum at the isobar of $0.1 \mathrm{MPa}$, but at higher pressures there is a maximum. The models are unable to predict this unusual thermodynamic behavior. The set $\mathrm{P} 4 \mathrm{C} \_3$ actually predicts $C_{P}^{r}$ minima but it predicts minima at the pressures $100 \mathrm{MPa}$ and $200 \mathrm{MPa}$, which are much higher than the pressure in which the actual minimum appears $(0.1 \mathrm{MPa})$. Additionally, the set $\mathrm{C} 4 \mathrm{C} \_1$ predicts maxima at high pressures but at higher temperatures (approximately 380-390 K) compared to the actual ones (approximately $320 \mathrm{~K}$ ). Unlike the density isobars (Figure 8), the calculated $C_{P}^{r}$ isobars with the various models and parameter sets do not have similar curvatures with each other.

Figure 10 shows experimental and calculated $C_{V}^{r}$ isobars of pure liquid water at four different pressures. The behavior of this property is not as complex as the behavior of $C_{P}^{r}$ and some of the calculated $C_{V}^{r}$ isobars are similar in curvature to the actual isobars. The set P4C_4 is able to predict this property with great accuracy especially at $400 \mathrm{MPa}$. The sets $\mathrm{C}_{4} \mathrm{C} \_1$ and $\mathrm{C} 3 \mathrm{~B} \_1$ predict maxima at much higher temperatures than the experimental values. For instance, at $200 \mathrm{MPa}$ the actual minimum resides at approximately $290 \mathrm{~K}$, the set C4C_1 predicts a maximum at approximately $390 \mathrm{~K}$ and $\mathrm{C} 3 \mathrm{~B} \_1$ predicts a maximum at approximately $550 \mathrm{~K}$. 

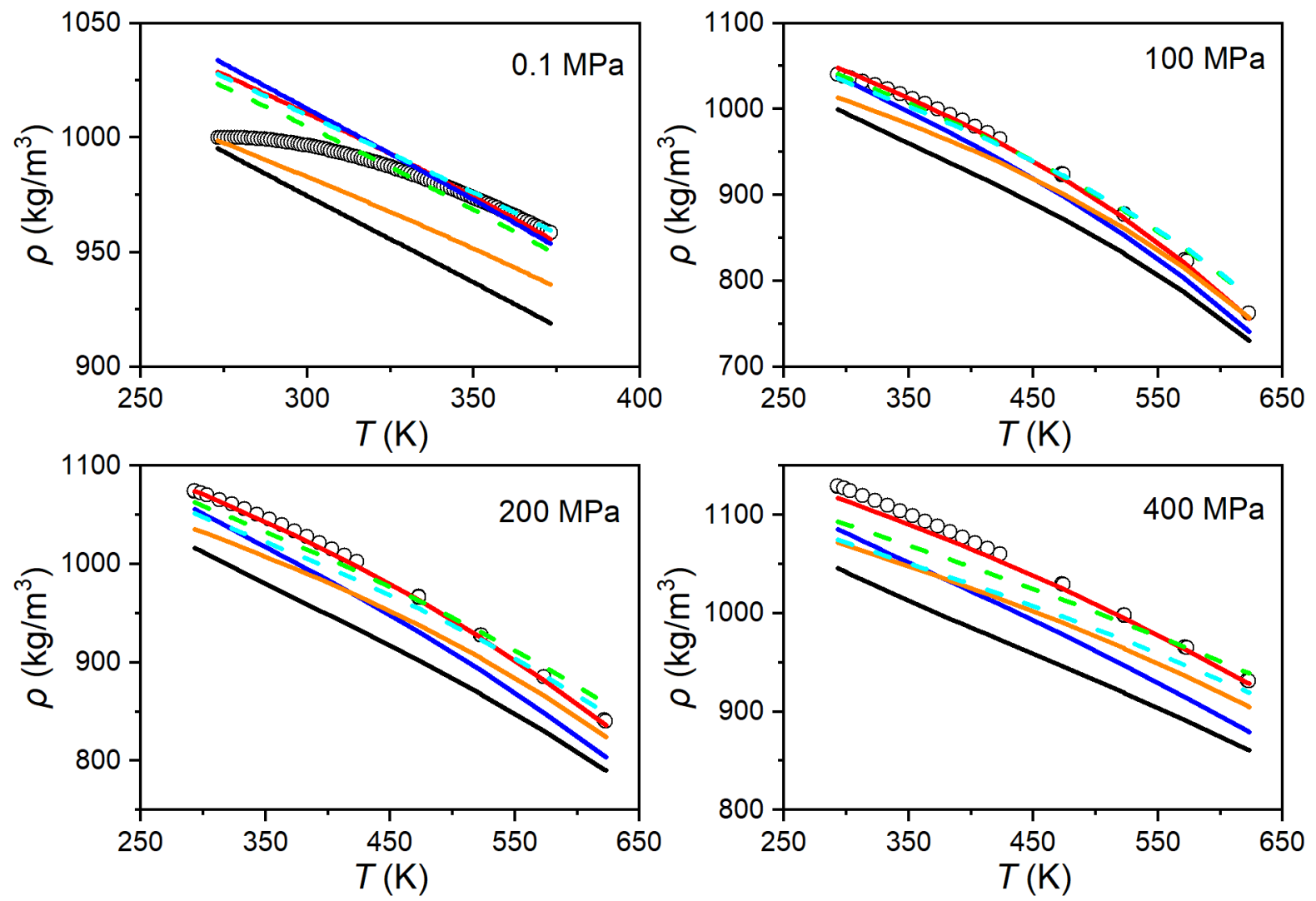

O $\operatorname{Exp} .-$ P2B_3-P4C_2 -P4C_3 P4C_5 - C4C_1 - - C3B_1

Figure 8. Experimental and calculated density isobars of pure liquid water with PC-SAFT and CPA with different parameter sets. The symbols represent the experimental data ${ }^{23-26}$ and the lines the calculated values with PC-SAFT and CPA.

Figure 11 shows experimental and calculated speed of sound isobars of pure liquid water at four different pressures. Speed of sound isobars display maxima at the different pressures. None of the models and parameter sets is able to predict these maxima and the calculated speed of sound continuously decreases when the temperature increases.

In short, none of the parameter sets investigated in this work was able to accurately predict any of the extrema. For $\rho$ and $u$, none of the parameter sets predict any extrema at all. For these two properties, it is as if the models treat water as a "normal liquid". For $C_{P}^{r}$ and $C_{V}^{r}$, some parameter sets are actually able to predict "some" extrema (i.e. at some conditions), but the predicted extrema are at different temperatures from the actual ones. It is also worth mentioning that the thermodynamic behavior of water changes significantly in relation to pressure. At higher pressures, the $\rho$ maximum disappears (Figure 3), $C_{P}^{r}$ minimum disappears (Figure 4 ), $C_{P}^{r}$ maxima and $C_{V}^{r}$ maxima start to appear (Figures 4 and 10). Meanwhile, the calculated properties with the models do not display such significant changes in relation to pressure. For example, the calculated $\rho$ isobars are linear at all pressure regions (Figure 8). Considering all of the above, it is likely that 
the models need substantial improvements if they are to predict (qualitatively and quantitatively) the anomalous behavior of water.
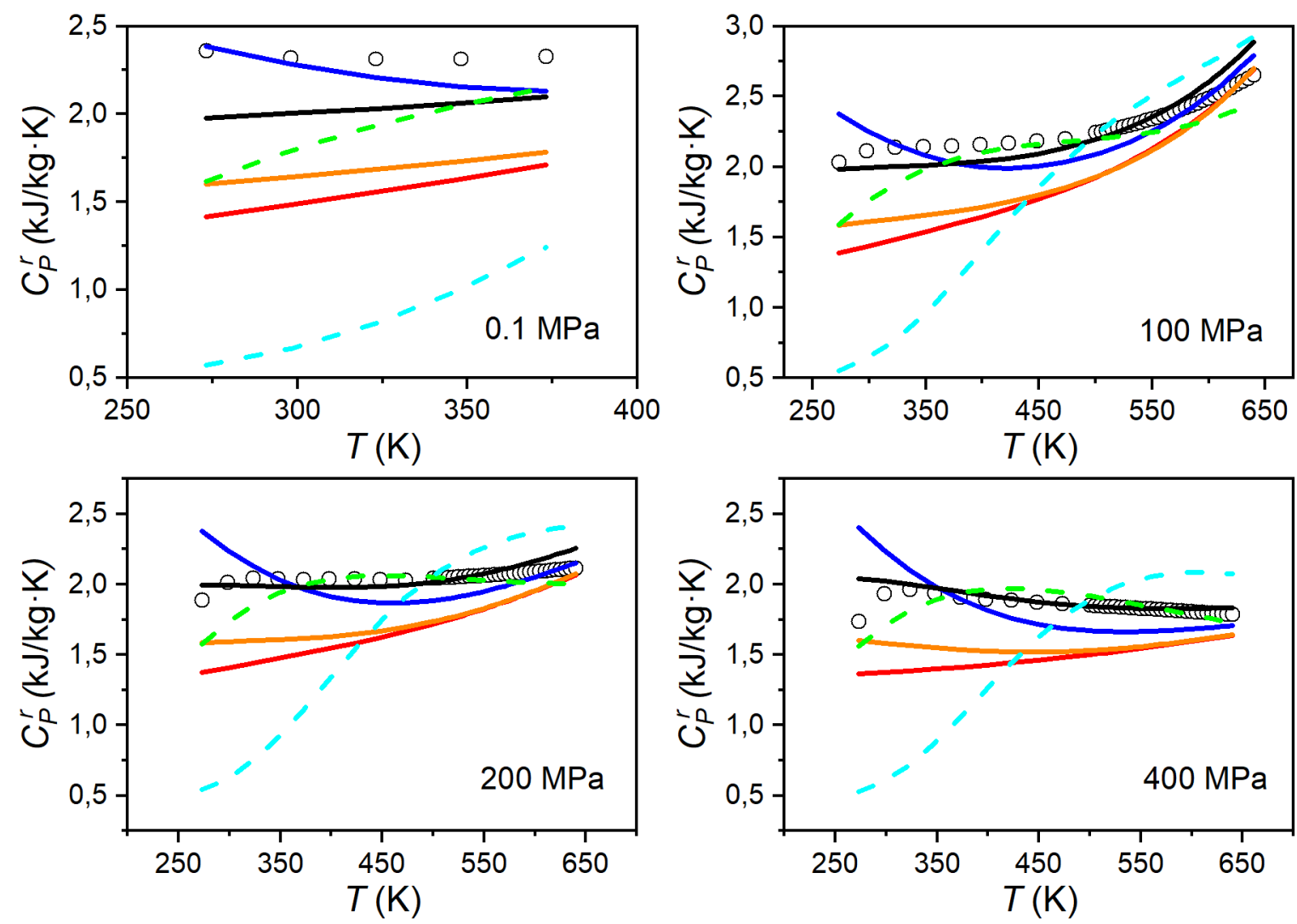

O Exp. $-P 2 B \_3-P 4 C \_2-P 4 C \_3-P 4 C \_5--C 4 C \_1--$ C3B_1

Figure 9. Experimental and calculated isobaric heat capacity isobars of pure liquid water with PCSAFT and CPA with different parameter sets. The symbols represent experimental data ${ }^{28}$ and results from the NIST model ${ }^{21}$ and lines represent the calculated values with PC-SAFT and CPA with different parameter sets.

5.3. Discussion on modifying molecular EOS for water. It is clear that PC-SAFT and CPA are inadequate for accurately describing water's unique thermodynamic behavior. It is likely that the parameters of the models could be fitted in order to capture the extremum of a thermodynamic property, but it is very unlikely that any of these models will be able to capture simultaneously all the extrema presented in this study. Therefore, these models should be modified in order to increase their accuracy. There are multiple directions that one could take for the modifications of these models and in fact there have been multiple studies that have modified molecular EOS in order to increase their accuracy. A short review of some of these studies follows with an emphasis on the prediction of anomalous behavior.

Palma et al. ${ }^{62}$ developed a new version of the CPA equation of state by modifying the energy parameter function $a(T)$. In specific, they added four more adjustable parameters to the expression of the new parameter function $a(T)$ of CPA. For this new version of CPA, the parametrization process has some restrictions. The constant parameter $a_{0}$ in the energy function and the co-volume 
parameter $b$ are fitted to the critical temperature and pressure of water. The other energy function parameters are fitted to vapor pressure data and the association parameters are fitted to heat capacity and vapor pressure data. In other words, the parameters were not fitted to liquid density data. They concluded that this modified version showed better results at various properties of water like vapor pressure, isobaric heat capacity and isochoric heat capacity. It did not provide better results for density, but their results were quite satisfactory (error of $1.10 \%$ ). In their evaluation they considered only saturated conditions for these properties and it is not completely clear whether this modified version is able to properly predict any of the extrema shown in our study. In their study, they also predicted phase equilibria of many water-containing systems (binary and ternary mixtures) with alkanes and alcohols for which the new modified CPA providing promising results. Regarding the solubility minimum of alkanes in the aqueous phase the modified CPA show a better trend compared to CPA results, but even though it shows better accuracy it does not predict a minimum.

As mentioned previously, PC-SAFT and CPA do not account for polar interactions between molecules. There have been various attempts to incorporate these interactions in molecular EOS. In one of these attempts, Karakatsani et al. ${ }^{63}$ extended the SAFT and PC-SAFT equations of state by adding an extra term that accounts for dipolar-dipolar interactions. The new equations of state are called PSAFT and PC-PSAFT and they based the new terms on the works of Nezbeda and Pavlíček ${ }^{64}$. What sets these models apart from other approaches is their simplicity, which makes them more suitable for engineering applications that involve multiple components. They applied both new models for a variety of different compounds including ketones, alcohols, water and more. The new models in general seem to provide quite satisfactory results for all the systems that were examined. However, it seems that these models are not able to predict the density maximum at saturated conditions since at these temperature regions the calculated values showed a different trend compared to the experimental data. Their study did not include any of the derivative properties mentioned here and so it is not certain whether these models will be able to predict any of the unusual characteristics that are present in the other properties.

Our models also do not account for hydrogen bond cooperativity. Marshall ${ }^{19}$ has developed a second order perturbation theory that incorporates hydrogen bonded cooperativity. To include this effect, they considered that the energy of the second hydrogen bond that a water molecule receives is higher if the first hydrogen bond is incident to a site with different functionality, i.e. if one is incident to a donor site and the other to an acceptor site. On the other hand, if both hydrogen bonds are incident to a site with the same functionality, they both have the same energy. The new association theory was able to predict monomer fraction data with exceptional accuracy without fitting to the data (parameter were fixed at logical values resulted from other studies). They used monomer fraction data from Luck and from Soper et al. ${ }^{65}$ for their evaluation. However, when the theory was implemented to an equation of state it required one parameter (association volume) to change and be adjusted to liquid density and vapor pressure data in order to provide satisfactory results. This new model performed well at predicting the density and vapor pressure, but not so well for the internal energy of vaporization. Furthermore, it was not able to predict the density maximum.

For the purposes of this study, it is important to note studies that have indeed managed to capture any of the anomalies that are present in water. Vega et al. ${ }^{66}$ have managed to predict solubility minima of hydrocarbons in the water rich phase. In their study, they used the soft-SAFT EOS. This equation does not account for polar interactions, it accounts for repulsive and dispersion forces and hydrogen bonding formation like PC-SAFT. The main differences are in the expression 
of the repulsive and dispersion forces and the reference system. The reference system is a LennardJones spherical fluid in contrast to a reference hard chain fluid used in PC-SAFT. In their study, they modeled water as a four-site molecule and they fitted the parameters to saturated liquid density and vapor pressure data and they introduced different parameter sets. The parameter set that was fitted at the temperature range $300-450 \mathrm{~K}$ was able to predict solubility minimum of ndecane with exceptional accuracy. For other hydrocarbons (n-octane, benzene, toluene) this set was able to predict solubility minima, but at different locations. The soft-SAFT equation of state showed considerably different qualitative results for the solubility of alkanes in water rich phase compared to the original SAFT, PC-SAFT and CPA since it was able to predict minima. Vega et al. ${ }^{66}$ suggest that this difference in the results is likely due to the fact that soft-SAFT uses a more realistic Helmholtz free energy expression for the repulsive and dispersion forces since they are included in the same term in contrast to the original SAFT. This model does not seem to be able to predict a density maximum.

One of the assumptions that take place in PC-SAFT (and many other SAFT variants) is that the structure of the fluid is not affected by hydrogen bonding. For water though that is not the case. Hydrogen bonds affect considerably water's structure and it has been reported that water undergoes structural transition to tetrahedral symmetry ${ }^{67-69}$. In recent studies from Marshall ${ }^{69,70}$ they managed to incorporate transition to tetrahedral structure in molecular EOS and they introduced associated reference perturbation theory (APT) and doubly associated reference perturbation theory (DAPT). Usually in molecular EOS for associating liquids a non associating reference fluid is used. In these new models the free energy contribution of the reference system is the sum of ideal gas, hard-sphere repulsion and association contributions. In other words the reference system is a hard sphere associating fluid. In $\mathrm{APT}^{70}$, the integral of the reference system pair correlation function is modified to incorporate the transition to tetrahedral symmetry. In specific, when all water molecules are fully hydrogen bonded the reference fluid shows tetrahedral coordination and when there is no hydrogen bonding the reference fluid shows the coordination of the standard hard-sphere reference fluid. It should be noted though that in APT the tetrahedral structure is taking into account during the calculation of the square-well attraction term. For the DAPT, Marshall ${ }^{71}$ incorporated the associating reference fluid integral to the TPT theory as well in order to incorporate the change to the association term as well. The results of these models are very promising. Both approaches provided satisfactory results for density, vapor pressure and heat of vaporization away from the critical temperature. It is extremely worth noting that these modifications are able to predict some anomalies. APT and DAPT are able to show minima in isothermal compressibility and isobaric heat capacity for temperatures, but only DAPT is able to predict the density maximum and the locus of the predicted maximum is very close to the actual one. It should be noted that DAPT considerably underpredicts the value of isobaric heat capacity at the minimum. Nevertheless, these changes are very promising for the prediction of water's complex thermodynamic behavior.

Another noteworthy study for the development of an improved EOS was conducted by Dufal et al. ${ }^{11}$. The main focus of the study was the SAFT-VR Mie EOS. One of the main characteristics of this model is that it is based on the Mie intermolecular potential, which is a generalized LennardJones potential with adjustable attractive and repulsive exponents that characterize the softness (or hardness) and the range of the interactions. When the radial distribution function of a hard sphere reference fluid was implemented in the association term, SAFT-VR Mie did not produce better results than SAFT-VR SW (another variant that uses a square-well reference fluid) even though SAFT-VR Mie has a more sophisticated theoretical treatment. It was concluded that the association 
term had to be modified to achieve better results. In specific, two association terms were developed, one with the radial distribution function of a Mie reference fluid and one with the radial distribution function of a Lennard-Jones reference fluid (similar to the work of Müller and Gubbins $^{13}$ ). The modified association terms could be incorporated in other SAFT variants as well, like SAFT, PC-SAFT, soft-SAFT and CPA. These modifications provided exceptional results for density, vapor pressure, Joule-Thomson coefficient, heat of vaporization and isobaric heat capacity of water with the most accurate being the first modification. Especially, for the singlephase region this approach was able to accurately predict these thermodynamic properties at supercritical conditions. However, most likely this approach does not predict the anomalies mentioned in our study. The trends of the calculated values for density and isobaric heat capacity appear to be slightly different from the trends of experimental values at low temperatures where many of the extrema appear. This small difference could be due to that water has a different structure than the reference fluid.

In short, there are multiple approaches for the development of more advanced molecular EOS. From this discussion, it seems that the structure of water plays a key role at the complex thermodynamic behavior. For this reason, APT and DAPT from Marshall ${ }^{71}$ have been the most effective at capturing water's anomalous behavior compared to the other studies presented here. These models are not able to capture the thermodynamic behavior of water with complete accuracy and therefore there is still room for improvement. It is possible that the incorporation of hydrogen bond cooperativity or polar interactions in DAPT would further improve its accuracy. Finally, all the studies discussed in this section have not examined all of the properties shown in our work. Thus, it is not certain whether these approaches can provide better results for the speed of sound or for isochoric heat capacity based on these studies. 

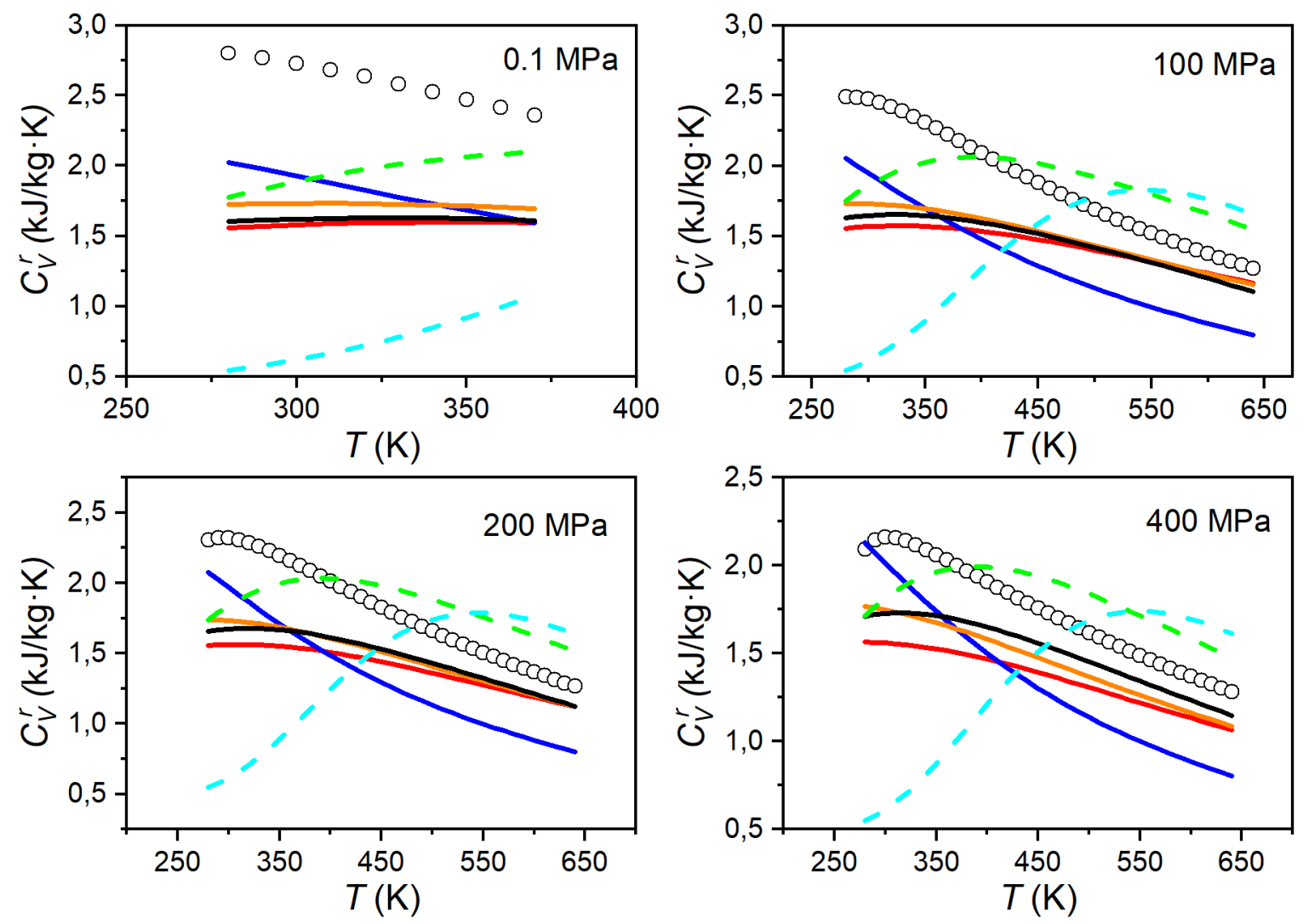

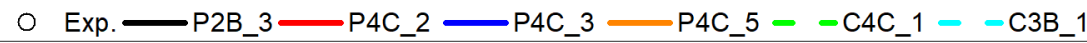

Figure 10. Experimental and calculated isochoric heat capacity isobars of pure liquid water. The symbols represent results from the NIST model ${ }^{21}$ and lines represent the calculated values with PC-SAFT and CPA with different parameter sets. 

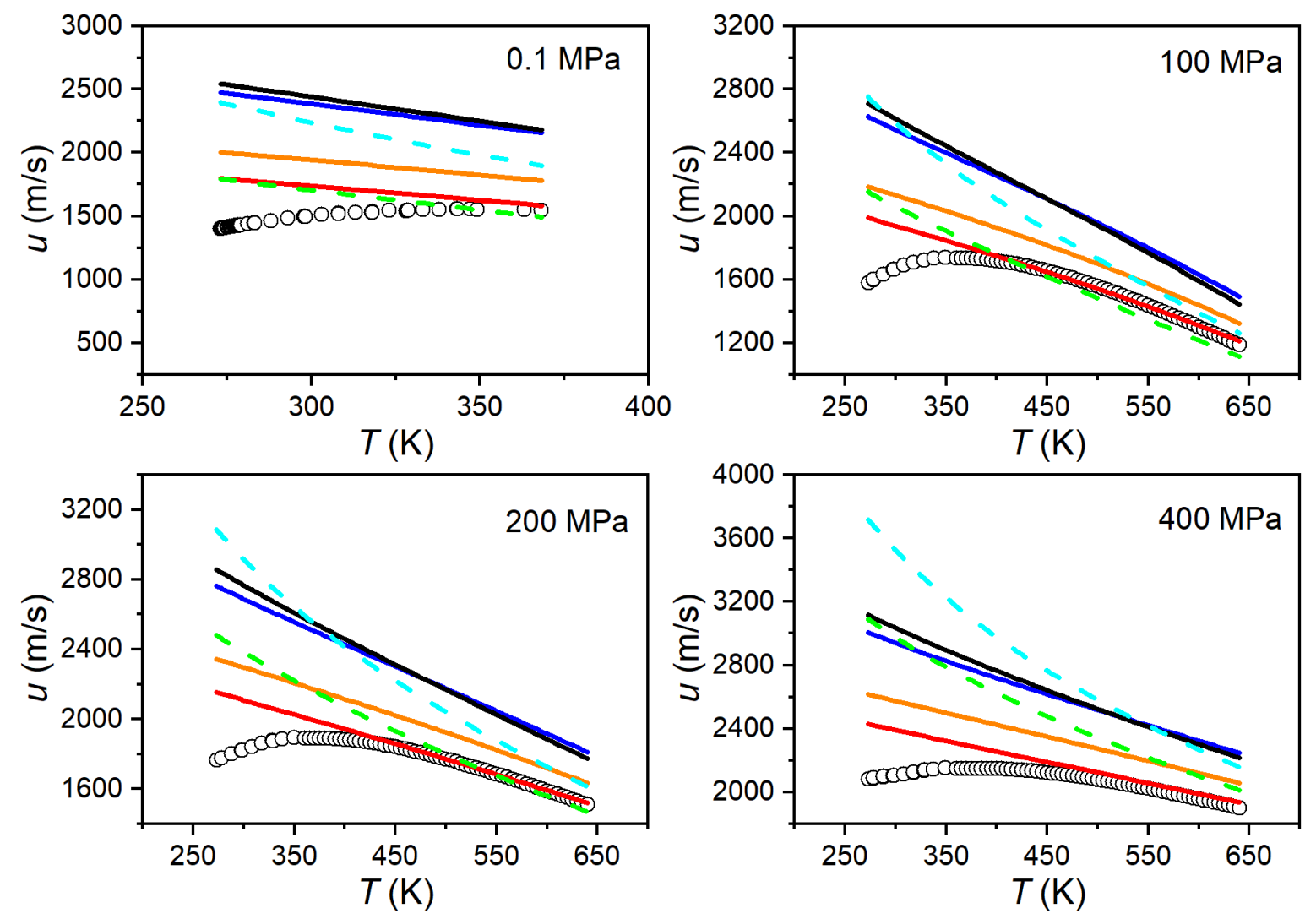

O Exp.—P2B_3-P4C_2 -P4C_3 P4C_5 - C4C_1 - - C3B_1

Figure 11. Experimental and calculated speed of sound isobars of pure liquid water. The symbols represent experimental data ${ }^{50-52}$ results from the NIST model ${ }^{21}$ and lines represent the calculated values with PC-SAFT and CPA with different parameter sets.

\section{Conclusions}

Two advanced equations of state, PC-SAFT and CPA, having an explicit term accounting for the hydrogen bonding effects have been considered for describing pure liquid water's thermodynamic properties. The properties that were studied in this work include vapor pressure, density, residual isobaric heat capacity, isochoric heat capacity and speed of sound. Several of them exhibit anomalous behavior with respect to temperature, not seen with other substances. Twelve PC-SAFT parameter sets and two CPA parameter sets from literature were compared. The majority of the parameter sets display satisfactory accuracy for the vapor pressure and they are able to predict the density within $13 \%$. In general, the parameter sets are less accurate in predicting the other (derivative) thermodynamic properties. Most of the parameter sets have been adjusted to vapor pressure and saturated liquid density data without considering any of the other properties. The physical meaning of the model parameters is not always retained during the parameter estimation. Some authors managed to assign physically justified values to some parameters. However, this process did not necessarily lead to more accurate predictions. 
Several of the water's thermodynamic properties studied here display an interesting "anomalous" behavior, which is not seen in other substances. Most of these properties show extrema when they are plotted against temperature, but none of the models was able to accurately predict these extrema. In fact, for the density and the speed of sound none of the parameter sets displayed any extrema.

These results indicate that the PC-SAFT and CPA equations of state are not able to properly model water's unusual thermodynamic behavior, despite providing an overall acceptable description of water properties over extensive temperature and pressure ranges. There are differences in the performances of the two models and of the many parameter sets considered, but overall we cannot name a "winner", neither among the models, nor among the parameter sets.

It can be mentioned that it could be possible for PC-SAFT or CPA to accurately predict the loci of (some) extrema, if parameters are adjusted to a specific property. Even so, it is unlikely that these models will be able to accurately predict all of the properties simultaneously with the same parameters based on the results of this study. Most likely, modifications will be needed in the underlying theories in order to account for other phenomena that are present in water and potentially being able to predict a wider range of thermodynamic properties of water, including anomalous properties.

There are multiple approaches for modifying these models. For instance, PC-SAFT and CPA do not account for polar interactions or hydrogen bon cooperativity. Both of these effects are known to be present in water. However, it should be noted that the inclusion of these phenomena do not necessarily improve the prediction of water's anomalous behavior. The most promising approach is to consider water's structure. The inclusion of water's transition to tetrahedral structure considerably improved the results especially from a qualitative point of view. Most likely, water's complex thermodynamic behavior is largely connected to its structure. Therefore, an accurate representation of water's structure could significantly improve the model's performance at predicting these behaviors.

\section{Acknowledgements}

The authors wish to thank the European Research Council (ERC) for funding this research under the European Union's Horizon 2020 research and innovation program (grant agreement No 832460), ERC Advanced Grant project "New Paradigm in Electrolyte Thermodynamics". The authors also wish to thank the Department of Chemical and Biochemical Engineering, Technical University of Denmark for funding this research.

\section{References}

1. Ball, P. Water — An Enduring Mystery. Nature 2008, 452, 291-292. 
2. Anomalous properties of water. http://www1.lsbu.ac.uk/water/water_anomalies.html (accessed May 26, 2020).

3. Aparicio-Martínez, S.; Hall, K., Phase Equilibria in Water Containing Binary Systems from Molecular Based Equations of State, Fluid Phase Equilib. 2007, 254, 112-125.

4. Valderrama, J. The State of the Cubic Equations of State. Ind. Eng. Chem. Res. 2003, 42, 1603-1618.

5. Wertheim, M. Fluids with Highly Directional Attractive Forces. I. Statistical Thermodynamics. J. Stat. Phys. 1984, 35, 19-34.

6. Wertheim, M. Fluids with Highly Directional Attractive Forces. II. Thermodynamic Perturbation Theory and Integral Equations. J. Stat. Phys. 1984, 35, 35-47.

7. Wertheim, M. Fluids with Highly Directional Attractive Forces. III. Multiple Attraction Sites. J. Stat. Phys. 1986, 42, 459-476.

8. Wertheim, M. Fluids with Highly Directional Attractive Forces. IV. Equilibrium Polymerization. J. Stat. Phys. 1986, 42, 477-492.

9. $\quad$ Liang, X.; Maribo-Mogensen, B.; Tsivintzelis, I.; Kontogeorgis, G. A Comment on Water's Structure using Monomer Fraction Data and Theories. Fluid Phase Equilib. 2016, 407, 2-6.

10. Luck, W. A Model of Hydrogen-Bonded Liquids. Angew. Chem., Int. Ed. Engl. 1980, 19, $28-41$.

11. Dufal, S.; Lafitte, T.; Haslam, A.; Galindo, A.; Clark, G.; Vega, C.; Jackson, G. The A in SAFT: Developing the Contribution of Association to the Helmholtz Free Energy within a Wertheim TPT1 Treatment of Generic Mie Fluids. Mol. Phys. 2015, 113, 948-984.

12. Maribo-Mogensen, B.; Kontogeorgis, G.; Thomsen, K. Modeling of Dielectric Properties of Complex Fluids with an Equation of State. J. Phys. Chem. B 2013, 117, 3389-3397.

13. Müller, E.; Gubbins, K. An Equation of State for Water from a Simplified Intermolecular Potential. Ind. Eng. Chem. Res. 1995, 34, 3662-3673.

14. Clark, G.; Haslam, A.; Galindo, A.; Jackson, G. Developing Optimal Wertheim-Like Models of Water for Use in Statistical Associating Fluid Theory (SAFT) and Related Approaches. Mol. Phys. 2006, 104, 3561-3581.

15. von Solms, N.; Michelsen, M.; Passos, C.; Derawi, S.; Kontogeorgis, G. Investigating Models for Associating Fluids using Spectroscopy. Ind. Eng. Chem. Res. 2006, 45, 53685374.

16. Grenner, A.; Schmelzer, J.; von Solms, N.; Kontogeorgis, G. Comparison of Two Association Models (Elliott-Suresh-Donohue And Simplified PC-SAFT) for Complex Phase Equilibria of Hydrocarbon-Water and Amine-Containing Mixtures. Ind. Eng. Chem. Res. 2006, 45, 8170-8179.

17. Kontogeorgis, G.; Tsivintzelis, I.; von Solms, N.; Grenner, A.; Bøgh, D.; Frost, M.; KnageRasmussen, A.; Economou, I. Use of Monomer Fraction Data in the Parametrization of Association Theories. Fluid Phase Equilib. 2010, 296, 219-229

18. Vega, L. F.; Llovell, F. Review and New Insights into the Application of Molecular-Based Equations of State to Water and Aqueous Solutions. Fluid Phase Equilib. 2016, 416, 150173.

19. Marshall, B. D. A Second Order Thermodynamic Perturbation Theory for Hydrogen Bond Cooperativity in Water. J. Chem. Phys. 2017, 146 (17) 
20. Mallamace, F.; Branca, C.; Broccio, M.; Corsaro, C.; Mou, C.; Chen, S. The Anomalous Behavior of the Density of Water in the Range $30 \mathrm{~K}<\mathrm{T}<373$ K. Proc. Natl. Acad. Sci. U. S. A. 2007, 104, 18387-18391.

21. Lemmon, E. W.; Linden, M. O.; Friend, D. G., Thermophysical Properties of Fluid Systems. In NIST Chemistry WebBook, NIST Standard Reference Database Number 69; Linstrom, P. J., Mallard, W. G., Eds.; National Institute of Standards and Technology: Gaithersburg, MD, 20899

22. Wagner, W.; Pruß, A. The IAPWS Formulation 1995 for the Thermodynamic Properties of Ordinary Water Substance for General and Scientific Use. J. Phys. Chem. Ref. Data 2002, 31, 387-535

23. Kell, G. Density, Thermal Expansivity, and Compressibility of Liquid Water from 0.Deg. to 150.Deg.. Correlations and Tables for Atmospheric Pressure and Saturation Reviewed and Expressed on 1968 Temperature Scale. J. Chem. Eng. Data 1975, 20, 97-105

24. Kell, G.; McLaurin, G.; Whalley, E. The PVT Properties of Water V. The Fluid to 1 KBAR at 350-500 Degrees $C$ and along the Saturation Line from 150 to 350 Degrees C. Philos. Trans. R. Soc., A 1985, 315, 235-246.

25. Hilbert, R.; Tödheide, K.; Franck, E. PVT Data for Water in the Ranges 20 to $600^{\circ} \mathrm{C}$ and 100 to 4000 Bar. Ber. Bunsenges. Phys. Chem. 1981, 85, 636-643.

26. Grindley, T.; Lind, J. PVT Properties of Water and Mercury. J. Chem. Phys. 1971, 54, 3983-3989.

27. Zhu, C.; Liu, X.; He, M.; Kontogeorgis, G.; Liang, X. Heat Capacities of Fluids: The Performance of Various Equations of State. J. Chem. Eng. Data 2020.

28. Lin, C.; Trusler, J. The speed of sound and derived thermodynamic properties of pure water at temperatures between (253 And 473) K and at pressures up to $400 \mathrm{MPa}$. J. Chem. Phys. 2012, 136, 094511

29. Brigham Young University. Design Institute for Physical Properties (DIPPR) Database: DIADEM Professional, Information and Data Evaluation Manager.; AIChE: New York, 2019.

30. Liang, X.; Tsivintzelis, I.; Kontogeorgis, G. Modeling Water Containing Systems with the Simplified PC-SAFT and CPA Equations of State. Ind. Eng. Chem. Res. 2014, 53, 1449314507.

31. Wei, S.; Shi, Z.; Castleman, A. Mixed Cluster Ions as a Structure Probe: Experimental Evidence for Clathrate Structure of $(\mathrm{H} 2 \mathrm{O}) 20 \mathrm{H}+$ and $(\mathrm{H} 2 \mathrm{O}) 21 \mathrm{H}+$. J. Chem. Phys. 1991, 94, 3268-3270.

32. Yakoumis, I.; Kontogeorgis, G.; Voutsas, E.; Hendriks, E.; Tassios, D. Prediction of Phase Equilibria in Binary Aqueous Systems Containing Alkanes, Cycloalkanes, and Alkenes with the Cubic-Plus-Association Equation of State. Ind. Eng. Chem. Res. 1998, 37, 41754182.

33. Economou, I.; Tsonopoulos, C. Associating Models and Mixing Rules in Equations of State for Water/Hydrocarbon Mixtures. Chem. Eng. Sci. 1997, 52, 511-525

34. Gross, J.; Sadowski, G. Application of the Perturbed-Chain SAFT Equation of State to Associating Systems. Ind. Eng. Chem. Res. 2002, 41, 5510-5515 
35. Cameretti, L.; Sadowski, G.; Mollerup, J. Modeling Of Aqueous Electrolyte Solutions with Perturbed-Chain Statistical Associated Fluid Theory. Ind. Eng. Chem. Res. 2005, 44, 33553362

36. Debye, P.; Hückel, E. Phys. Z. 1923, 9, 185-206.

37. Diamantonis, N.; Economou, I. Evaluation of Statistical Associating Fluid Theory (SAFT) and Perturbed Chain-SAFT Equations of State for the Calculation of Thermodynamic Derivative Properties of Fluids Related to Carbon Capture and Sequestration. Energy Fuels 2011, 25, 3334-3343

38. Kontogeorgis, G. M.; Yakoumis, I. V.; Meijer, H.; Hendriks, E. M.; Moorwood, T. Multicomponent Phase Equilibrium Calculations for Water- Methanol - Alkane Mixtures. Fluid Phase Equilib. 1999, 158 - 160, 201.

39. Kontogeorgis, G.; Michelsen, M.; Folas, G.; Derawi, S.; von Solms, N.; Stenby, E. Ten Years with the CPA (Cubic-Plus-Association) Equation of State. Part 1. Pure Compounds and Self-Associating Systems. Ind. Eng. Chem. Res. 2006, 45, 4855-4868

40. Xantheas, S. Cooperativity and Hydrogen Bonding Network in Water Clusters. Chem. Phys. 2000, 258, 225-231.

41. Chapman, W. G.; Gubbins, K. E.; Jackson, G.; Radosz, M. SAFT Equation-of-state solution model for associating fluids. Fluid Phase Equilib. 1989, 52, 31.

42. Chapman, W. G.; Gubbins, K. E.; Jackson, G.; Radosz, M. New reference equation of state for associating liquids. Ind. Eng. Chem. Res. 1990, 29, 1709

43. Gross, J.; Sadowski, G. Perturbed-Chain SAFT: An Equation of State Based on a Perturbation Theory for Chain Molecules. Ind. Eng. Chem. Res. 2001, 40, 1244-1260.

44. Barker, J. A.; Henderson, D. Perturbation Theory and Equation of State for Fluids: The Square Well Potential. J. Chem. Phys. 1967, 47, 2856.

45. Barker, J. A.; Henderson, D. Perturbation Theory and Equation of State for Fluids. II. A Successful Theory of Liquids. J. Chem. Phys. 1967, 47, 4714

46. Kontogeorgis, G. M.; Folas, G. K. Thermodynamic Models for Industrial Applications From Classical and Advanced Mixing Rules to Association Theories; John Wiley and Sons: New York, 2010.

47. von Solms, N.; Michelsen, M. L.; Kontogeorgis, G. M. Computational and physical performance of a modified PC-SAFT equation of state for highly asymmetric systems and associating mixtures. Ind. Eng. Chem. Res. 2003, 42, 1098

48. Kontogeorgis, G.; Voutsas, E.; Yakoumis, I.; Tassios, D. An Equation of State for Associating Fluids. Ind. Eng. Chem. Res. 1996, 35, 4310-4318.

49. Sun, L.; Kontogeorgis, G.; von Solms, N.; Liang, X. Modeling of Gas Solubility using the Electrolyte Cubic Plus Association Equation of State. Ind. Eng. Chem. Res. 2019, 58, 17555-17567.

50. Hidalgo Baltasar, E.; Taravillo, M.; Baonza, V.; Sanz, P.; Guignon, B. Speed of Sound in Liquid Water from (253.15 to 348.15) K and Pressures from (0.1 to 700) MPa. J. Chem. Eng. Data 2011, 56, 4800-4807.

51. Del Grosso, V. Sound Speed in Pure Water and Sea Water. J. Acoust. Soc. Am. 1970, 47, 947-949

52. Alexandrov A. A.; Larkin D. K. Teploenergetika 1976, 23, 75 
53. Osborne, N.; Stimson, H.; Fiock, E.; Ginnings, D. The Pressure of Saturated Water Vapor in the Range $100{ }^{\circ} \mathrm{C}$ to $374{ }^{\circ} \mathrm{C}$. Bur. Stand. J. Res. 1933, 10, 155.

54. Hanafusa, H.; Kawai, K.; Sato, H.; Uematsu, M.; Watanabe, K. PVT Measurements and Critical Parameters of Water. MRS Online Proc. Libr. 1983, 22.

55. Guildner, L.; Johnson, D.; Jones, F. Vapor Pressure of Water at its Triple Point. J. Res. Natl. Bur. Stand., Sect. A 1976, 80A, 505.

56. Stimson, H. Some Precise Measurements of the Vapor Pressure of Water in the Range from 25 to 100 C. J. Res. Natl. Bur. Stand., Sect. A 1969, 73A, 493.

57. Morita, T.; Sato, H.; Uematsu, M.; Watanabe, K. PVT Properties and Vapor-Pressures of Ordinary Water Substance in the Critical Region. Phys. A (Amsterdam, Neth.) 1989, 156, 436-453.

58. Osborne, N.; Stimson, H.; Ginnings, D. Thermal Properties of Water and Steam from 100 to $374^{\circ}$ C. Bur. Stand. J. Res. 1937, 18, 389-447

59. Smith, L.; Keyes, F. The Volume of Unit Mass of Liquid Water and their Correlation as a Function of Pressure and Temperature. Part III. Stem Research Program. Proc. Am. Acad. Arts Sci. 1934, 69, 285-314.

60. Sirota, A. M., Inzh.-Fiz. Zh. 1963, 6, 52

61. Chávez, M.; Sosa, V.; Tsumura, R. Speed of Sound in Saturated Pure Water. J. Acoust. Soc. Am. 1985, 77, 420-423

62. Palma, A.; Queimada, A.; Coutinho, J. Improved Prediction of Water Properties and Phase Equilibria with a Modified Cubic Plus Association Equation of State. Ind. Eng. Chem. Res. 2017, 56, 15163-15176.

63. Karakatsani, E.; Spyriouni, T.; Economou, I. Extended Statistical Associating Fluid Theory (SAFT) Equations of State for Dipolar Fluids. AIChE J. 2005, 51, 2328-2342.

64. Nezbeda, I.; Pavlíček, J. Application of Primitive Models of Association: A Simple Theoretical Equation of State Of Water. Fluid Phase Equilib. 1996, 116, 530-536.

65. Soper, A.; Bruni, F.; Ricci, M. Site-Site Pair Correlation Functions of Water From 25 to $400^{\circ} \mathrm{C}$ : Revised Analysis of New and Old Diffraction Data. J. Chem. Phys. 1997, 106, 247-254.

66. Vega, L.; Llovell, F.; Blas, F. Capturing the Solubility Minima of n-Alkanes in Water by Soft-SAFT. J. Phys. Chem. B 2009, 113, 7621-7630.

67. Gallo, P.; Amann-Winkel, K.; Angell, C. A.; Anisimov, M. A.; Caupin, F.; Chakravarty, C.; Lascaris, E.; Loerting, T.; Panagiotopoulos, A. Z.; Russo, J.; Sellberg, J. A.; Stanley, H. E.; Tanaka, H.; Vega, C.; Xu, L.; Pettersson, L. G. M. Water: A Tale of Two Liquids. Chem. Rev. 2016, 116 (13), 7463-7500.

68. Errington, J.; Debenedetti, P. Relationship between Structural Order and the Anomalies of Liquid Water. Nature 2001, 409, 318-321.

69. Nilsson, A.; Pettersson, L. The Structural Origin of Anomalous Properties of Liquid Water. Nat. Commun. 2015, 6.

70. Marshall, B. Perturbation Theory for Water with an Associating Reference Fluid. Phys. Rev. E 2017, 96.

71. Marshall, B. A Doubly Associated Reference Perturbation Theory for Water. Fluid Phase Equilib. 2019, 500, 112252 
For Table of Contents Only
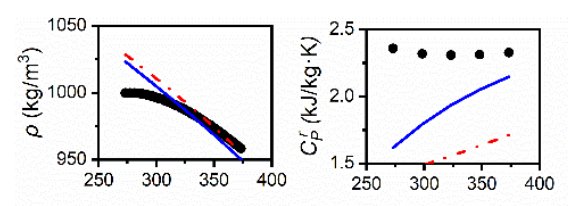

Association models:

Accurate for many

substances
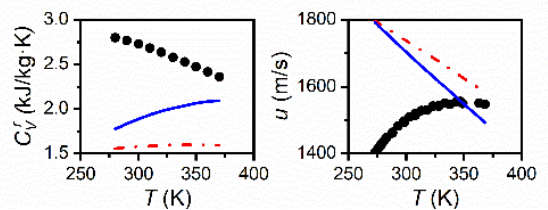

Cannot predict water's

anomalies

Improvements in the theories are needed

(K) 